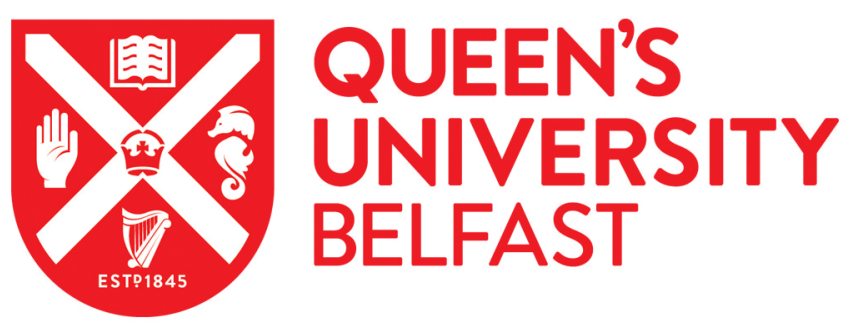

\title{
Gravitational wave signals from 3D neutrino hydrodynamics simulations of core-collapse supernovae
}

Andresen, H., Mueller, B., Mueller, E., \& Janka, H-T. (2017). Gravitational wave signals from 3D neutrino hydrodynamics simulations of core-collapse supernovae. Monthly Notices of the Royal Astronomical Society, 468(2), 2032-2051. https://doi.org/10.1093/mnras/stx618

Published in:

Monthly Notices of the Royal Astronomical Society

Document Version:

Publisher's PDF, also known as Version of record

Queen's University Belfast - Research Portal:

Link to publication record in Queen's University Belfast Research Portal

Publisher rights

Copyright 2017 The Authors. Published by Oxford University Press on behalf of the Royal Astronomical Society.

This work is made available online in accordance with the publisher's policies. Please refer to any applicable terms of use of the publisher.

\section{General rights}

Copyright for the publications made accessible via the Queen's University Belfast Research Portal is retained by the author(s) and / or other copyright owners and it is a condition of accessing these publications that users recognise and abide by the legal requirements associated with these rights.

Take down policy

The Research Portal is Queen's institutional repository that provides access to Queen's research output. Every effort has been made to ensure that content in the Research Portal does not infringe any person's rights, or applicable UK laws. If you discover content in the Research Portal that you believe breaches copyright or violates any law, please contact openaccess@qub.ac.uk. 


\title{
Gravitational wave signals from 3D neutrino hydrodynamics simulations of core-collapse supernovae
}

\author{
H. Andresen, ${ }^{1,2 \star}$ B. Müller, ${ }^{3,4}$ E. Müller ${ }^{1 \star}$ and H.-Th. Janka ${ }^{1}$ \\ ${ }^{1}$ Max-Planck-Institut für Astrophysik, Karl-Schwarzschild-Str. 1, D-85748 Garching, Germany \\ ${ }^{2}$ Physik Department, Technische Universität München, James-Franck-Str. 1, D-85748 Garching, Germany \\ ${ }^{3}$ Astrophysics Research Centre, School of Mathematics and Physics, Queen's University Belfast, Belfast BT7 1NN, UK \\ ${ }^{4}$ Monash Centre for Astrophysics, School of Physics and Astronomy, Building 79P, Monash University, VIC 3800, Australia
}

Accepted 2017 March 10. Received 2017 March 10; in original form 2016 July 15

\begin{abstract}
We present gravitational wave $(\mathrm{GW})$ signal predictions from four 3D multigroup neutrino hydrodynamics simulations of core-collapse supernovae of progenitors with 11.2, 20 and $27 \mathrm{M}_{\odot} . \mathrm{GW}$ emission in the pre-explosion phase strongly depends on whether the post-shock flow is dominated by the standing accretion shock instability (SASI) or convection and differs considerably from 2D models. SASI activity produces a strong signal component below $250 \mathrm{~Hz}$ through asymmetric mass motions in the gain layer and a non-resonant coupling to the protoneutron star (PNS). Both convection- and SASI-dominated models show GW emission above $250 \mathrm{~Hz}$, but with considerably lower amplitudes than in 2D. This is due to a different excitation mechanism for high-frequency $l=2$ motions in the PNS surface, which are predominantly excited by PNS convection in 3D. Resonant excitation of high-frequency surface g modes in $3 \mathrm{D}$ by mass motions in the gain layer is suppressed compared to $2 \mathrm{D}$ because of smaller downflow velocities and a lack of high-frequency variability in the downflows. In the exploding $20 \mathrm{M}_{\odot}$ model, shock revival results in enhanced low-frequency emission due to a change of the preferred scale of the convective eddies in the PNS convection zone. Estimates of the expected excess power in two frequency bands suggest that second-generation detectors will only be able to detect very nearby events, but that third-generation detectors could distinguish SASI- and convection-dominated models at distances of $\sim 10 \mathrm{kpc}$.
\end{abstract}

Key words: gravitational waves - hydrodynamics - instabilities - supernovae: general.

\section{INTRODUCTION}

Despite impressive progress during recent years, the explosion mechanism powering core-collapse supernovae is still not fully understood. For ordinary supernovae with explosion energies up to $\sim 10^{51} \mathrm{erg}$, the prevailing theory is the delayed neutrino-driven mechanism (see Janka 2012; Burrows 2013 for current reviews). In this scenario, the shock wave formed during the rebound (bounce) of the inner core initially stalls and only propagates out to a radius of $\sim 150 \mathrm{~km}$. The energy needed to revitalize the shock is provided by the partial re-absorption of neutrinos emitted from the proto-neutron star (PNS).

Hydrodynamical instabilities operating behind the stalled shock front have been found to be crucial for the success of this scenario as they help to push the shock further out by generating large Reynolds stresses (or 'turbulent pressure', see Burrows, Hayes

^E-mail: haakoan@mpa-garching.mpg.de (HA);

ewald@MPA-Garching.MPG.DE (EM)
\& Fryxell 1995; Murphy, Dolence \& Burrows 2013; Couch \& Ott 2015; Müller \& Janka 2015) and transporting neutrino-heated material out from the gain radius, which then allows the material to be exposed to neutrino heating over a longer 'dwell time' (Buras et al. 2006b; Murphy \& Burrows 2008b). Moreover, if the instabilities lead to the formation of sufficiently large high-entropy bubbles, the buoyancy of these bubbles can become high enough to allow them to rise and expand continuously (Thompson 2000; Dolence et al. 2013; Fernández 2015).

Two such instabilities have been identified in simulations, namely the more familiar phenomenon of convection driven by the unstable entropy gradient arising due to neutrino heating (Bethe 1990; Herant et al. 1994; Burrows et al. 1995; Janka \& Müller 1996; Müller \& Janka 1997), and the so-called standing accretion shock instability (SASI), which manifests itself in large-scale sloshing and spiral motions of the shock (Blondin, Mezzacappa \& DeMarino 2003; Blondin \& Mezzacappa 2006; Ohnishi, Kotake \& Yamada 2006; Foglizzo et al. 2007, 2015; Ohnishi et al. 2008; Scheck et al. 2008; Guilet \& Foglizzo 2012). After initial setbacks in three-dimensional (3D) supernova modelling, we are now starting to see the emergence 
of the first generation of successful 3D simulations of explosions with three-flavour multigroup neutrino transport, culminating in the recent models of the Garching and Oak Ridge groups (Lentz et al. 2015; Melson, Janka \& Marek 2015a; Melson et al. 2015b) with their rigorous treatment of the transport and neutrino microphysics in addition to many more obtained with more approximate transport schemes, as for example the studies of Takiwaki, Kotake \& Suwa (2012, 2014), Müller (2015) and Roberts et al. (2016). ${ }^{1}$

Our means to validate these numerical models by observations are limited. Classical photon-based observations of supernovae and their remnants (e.g. mixing in the envelope, see Wongwathanarat, Müller \& Janka 2015 and references therein; pulsar kicks, Scheck et al. 2006; Wongwathanarat, Janka \& Müller 2010; Nordhaus et al. 2012; Wongwathanarat, Janka \& Müller 2013) provide only indirect constraints on the workings of the hydrodynamic instabilities in the inner engine of a supernova. For a nearby, Galactic supernova event, messengers from the core in the form of neutrinos and gravitational waves (GWs) could furnish us with a direct glimpse at the engine. Neutrinos, for example, could provide a smoking gun for SASI activity through fast temporal variations (Marek, Janka \& Müller 2009; Lund et al. 2010; Brandt et al. 2011; Tamborra et al. 2013, 2014a; Müller \& Janka 2014) and could even allow a time-dependent reconstruction of the shock trajectory (Müller \& Janka 2014).

Likewise, a detection of GWs could potentially help to unveil the multidimensional effects operating in the core of a supernova. The signal from the collapse and bounce of rapidly rotating iron cores and triaxial instabilities in the early post-bounce phase has long been studied in 2D (i.e. under the assumption of axisymmetry) and 3D (e.g. Dimmelmeier et al. 2007, 2008; Ott et al. 2007; Scheidegger et al. 2008; Abdikamalov et al. 2010). Understanding the GW signal generated by convection and the SASI in the more generic case of slow or negligible rotation has proved more difficult due to a more stochastic nature of the signal. During the recent years, however, a coherent picture of GW emission has emerged from parametrized models (Murphy, Ott \& Burrows 2009) and first-principle simulations of supernova explosions in 2D (Marek, Janka \& Müller 2009; Müller, Janka \& Marek 2013): the models typically show an early, low-frequency signal with typical frequencies of $\sim 100 \mathrm{~Hz}$ arising from shock oscillations that are seeded by prompt convection (Marek et al. 2009; Murphy et al. 2009; Yakunin et al. 2010; Müller et al. 2013; Yakunin et al. 2015). This signal component is followed by a high-frequency signal with stochastic amplitude modulations that is generated by forced oscillatory motions in the convectively stable neutron star surface layer (Marek et al. 2009; Murphy et al. 2009; Müller et al. 2013) with typical frequencies of $300 \ldots 1000 \mathrm{~Hz}$ that closely trace the Brunt-Väisäla frequency in this region (Müller et al. 2013). Prior to the explosion, these oscillations, tentatively identified as $l=2$ surface $\mathrm{g}$ modes by Müller et al. (2013), are primarily driven by the downflows imping-

\footnotetext{
${ }^{1}$ Takiwaki et al. $(2012,2014)$ employ the isotropic diffusion source approximation (Liebendörfer, Whitehouse \& Fischer 2009) and use further approximations to treat heavy lepton neutrinos. Takiwaki et al. (2014) employ a leakage scheme to account for heavy lepton neutrinos and Takiwaki et al. (2012) neglect the effect of these neutrinos altogether. Müller (2015) utilizes the stationary fast multigroup transport scheme of Müller \& Janka (2015), which at high optical depths solves the Boltzmann equation in a two-stream approximation and matches the solution to an analytic variable Eddington factor closure at low optical depths. Roberts et al. (2016) employ a full 3D two-moment (M1) solver in general relativistic simulations, but ignore velocity-dependent terms.
}

ing on to the neutron star, whereas PNS convection takes over as the forcing agent a few hundred milliseconds after shock revival as accretion dies down. This high-frequency contribution dominates the energy spectrum and the total energy emitted in GWs can reach $\sim 10^{46}$ erg (Müller et al. 2013; Yakunin et al. 2015).

Since 3D supernova models have proved fundamentally different to $2 \mathrm{D}$ models in many respects, it stands to reason that much of what we have learned about GW emission from first-principle 2D models will need to be revised. In 2D, the inverse turbulent cascade (Kraichnan 1967) facilitates the emergence of large-scale flow structures also in convectively dominated models and helps to increase the kinetic energy in turbulent fluid motions in the post-shock region (Hanke et al. 2012). Furthermore, accretion downflows impact the PNS with much higher velocities in 2D than in 3D (Melson et al. 2015a) due to the inverse turbulent cascade and the stronger inhibition of Kelvin-Helmholtz instabilities at the interface of supersonic accretion downflows (Müller 2015). In the SASI-dominated regime, on the other hand, the additional dimension allows the development of the spiral mode (Blondin \& Mezzacappa 2007; Blondin \& Shaw 2007; Fernández 2010) in 3D, which can store more non-radial kinetic energy than pure sloshing motions in $2 \mathrm{D}$ (Hanke et al. 2013; Fernández 2015), contrary to earlier findings of Iwakami et al. (2008). Such far-reaching differences between 2D and 3D cannot fail to have a significant impact on the GW signal.

While the impact of 3D effects on the GW signals from the postbounce phase has been investigated before, all available studies have relied on a rather approximate treatment of neutrino heating and cooling such as simple light-bulb models (Müller \& Janka 1997; Kotake et al. 2009; Kotake, Iwakami-Nakano \& Ohnishi 2011), grey neutrino transport (Fryer, Holz \& Hughes 2004; Müller, Janka \& Wongwathanarat 2012a) or a partial implementation of the isotropic diffusion source approximation of Liebendörfer et al. (2009) in the works of Scheidegger et al. (2008, 2010), which were also limited to the early post-bounce phase. Arguably, none of these previous studies have as yet probed precisely the regimes encountered by the best current 3D simulations (e.g. the emergence of a strong SASI spiral mode) and therefore cannot be relied upon for quantitative predictions of GW amplitudes and spectra, which are extremely sensitive to the nature of hydrodynamic instabilities, the neutrino heating and the contraction of the PNS.

In this paper, we present GW waveforms of the first few hundred milliseconds of the post-bounce phase computed from 3D models with multigroup neutrino transport. Waveforms have been analysed for four supernova models of progenitors with zero-age main sequence (ZAMS) masses of 11.2, 20 (for which we study an exploding and a non-exploding simulation) and $27 \mathrm{M}_{\odot}$. With four simulations based on these three different progenitors, we cover both the convective regime $\left(11.2 \mathrm{M}_{\odot}\right)$ and the SASI-dominated regime (20 and $27 \mathrm{M}_{\odot}$ ). Our aim in studying waveforms from these progenitors is twofold: on the one hand, we shall attempt to unearth the underlying hydrodynamical phenomena responsible for the GW emission in different regions of the frequency spectrum during different phases of the evolution. We shall also compare the GW emission in 3D and 2D models, which will further illuminate dynamical differences between 2D and 3D. Furthermore, with 3D models now at hand, we are in a position to better assess the detectability of GWs from the post-bounce phase in present and future instruments than with $2 \mathrm{D}$ models affected by the artificial constraint of axisymmetry.

One of our key findings is that the GW signal from SASIdominated models is clearly differentiated from convectiondominated model by strong emission in a low-frequency band 
around $100 \ldots 200 \mathrm{~Hz}$. Very recently, Kuroda, Kotake \& Takiwaki (2016) also studied the GW signal features (in models using grey neutrino transport) during phases of SASI activity for a $15 \mathrm{M}_{\odot}$ star, comparing results for three different nuclear equations of state. Going beyond Kuroda et al. (2016), we clarify why this signature has not been seen in 2D models and point out that the hydrodynamic processes underlying this low-frequency signal are quite complex and seem to require a coupling of SASI motions to deeper layers inside the PNS. Moreover, we show that broad-band low-frequency GW emission can also occur after the onset of the explosion and is therefore not an unambiguous signature of the SASI. We also provide a more critical assessment of the detectability of this new signal component, suggesting that it may only be detectable with secondgeneration instruments like Advanced LIGO for a very nearby event at a distance of $2 \mathrm{kpc}$ or less.

Our paper is structured as follows: we first give a brief description of the numerical setup and the extraction of GWs in Section 2. In Section 3, we present a short overview of the GW waveforms and then analyse the hydrodynamical processes contributing to different parts of the spectrum in detail. We also compare our results to recent studies based on 2D first-principle models. In Section 4, we discuss the detectability of the predicted GW signal from our three progenitors by Advanced LIGO (The LIGO Scientific Collaboration et al. 2015) and by the Einstein Telescope (Sathyaprakash et al. 2012) as next-generation instrument. We also comment on possible inferences from a prospective $\mathrm{GW}$ detection. Our conclusions and a summary of open questions for future research are presented in Section 5.

\section{SIMULATION SETUP}

\subsection{Numerical methods}

The simulations were performed with the PROMETHEUS-VERTEX code (Rampp \& Janka 2002; Buras et al. 2006a). The Newtonian hydrodynamics module PROMETHEus (Fryxell, Arnett \& Müller 1991; Müller, Fryxell \& Arnett 1991) features a dimensionally split implementation of the piecewise parabolic method of Colella \& Woodward (1984) in spherical polar coordinates $(r, \theta, \varphi)$. Selfgravity is treated using the monopole approximation, and the effects of general relativity are accounted for in an approximate fashion by means of a pseudo-relativistic effective potential (case A of Marek et al. 2006). The neutrino transport module VERTEX (Rampp \& Janka 2002) solves the energy-dependent two-moment equations for three neutrino species $\left(v_{e}, \bar{v}_{e}\right.$, and a species $v_{X}$ representing all heavy flavour neutrinos) using a variable Eddington factor technique. The 'ray-by-ray-plus' approximation of Buras et al. (2006a) is applied to make the multi-D transport problem tractable. In the high-density regime, the nuclear equation of state $(\mathrm{EoS})$ of Lattimer \& Swesty (1991) with a bulk incompressibility modulus of nuclear matter of $K=220 \mathrm{MeV}$ has been used in all cases.

\subsection{Supernova models}

\subsubsection{D models}

We study four 3D models based on three solar-metallicity progenitor stars with ZAMS masses of 11.2 (Woosley, Heger \& Weaver 2002), 20 (Woosley \& Heger 2007) and $27 \mathrm{M}_{\odot}$ (Woosley et al. 2002). An initial grid resolution of $400 \times 88 \times 176$ zones in $r, \theta$ and $\varphi$ was used for the $3 \mathrm{D}$ models, and more radial grid zones were added during the simulations to maintain sufficient resolution around the
PNS surface. The innermost $10 \mathrm{~km}$ were simulated in spherical symmetry to avoid excessive limitations on the time step when applying a spherical polar grid.

(i) s11.2: model s11.2 (Tamborra et al. 2014b) is based on the solar-metallicity $11.2 \mathrm{M}_{\odot}$ progenitor of Woosley et al. (2002). This model exhibits transient shock expansion after the infall of the $\mathrm{Si} / \mathrm{O}$ shell interface, but falls slightly short of an explosive runaway. After the average shock radius reaches a maximum of $\approx 250 \mathrm{~km}$ at a time of $\approx 200 \mathrm{~ms}$ after bounce, the shock recedes and shock revival is not achieved by the end of the simulation $352 \mathrm{~ms}$ after core bounce. The post-shock region is dominated by buoyancy-driven convection; because of the large shock radius no growth of the SASI is observed. The convective bubbles remain of moderate scale: even during the phase of strongest shock expansion around $\sim 200 \mathrm{~ms}$ after bounce when the shock deformation is most pronounced and the kinetic energy in convection motions reaches its peak value, the bubbles subtend angles of no more than $\lesssim 60^{\circ}$.

(ii) s20: model s20 is based on the $20 \mathrm{M}_{\odot}$ solar-metallicity progenitor of Woosley \& Heger (2007) and has been discussed in greater detail in Tamborra et al. (2013, 2014a), where quasi-periodic modulations of the neutrino emission were analysed and traced back to SASI-induced variations of the mass-accretion flow to the PNS. No explosion is observed by the end of the simulation $421 \mathrm{~ms}$ post-bounce. There is an extended phase of strong SASI activity (dominated by the spiral mode) between 120 and $280 \mathrm{~ms}$ after core bounce. After a period of transient shock expansion following the infall of the $\mathrm{Si} / \mathrm{O}$ shell interface, SASI activity continues, but the kinetic energy in the SASI remains considerably smaller than during its peak between 200 and $250 \mathrm{~ms}$.

(iii) s20s: this model is based on the same $20 \mathrm{M}_{\odot}$ progenitor as s20, but a non-zero contribution from strange quarks to the axial-vector coupling constant, $g_{\mathrm{a}}^{\mathrm{s}}=-0.2$, from neutral-current neutrino-nucleon scattering was assumed (Melson et al. 2015b). This modification of the neutrino interaction rates results in a successful explosion (Melson et al. 2015b). Shock revival sets in around $300 \mathrm{~ms}$ after bounce. Prior to shock revival, the post-shock flow is dominated by large-scale SASI sloshing motions between 120 and $280 \mathrm{~ms}$ post-bounce. By the end of the simulation $528 \mathrm{~ms}$ postbounce, the average shock radius is $\approx 1000 \mathrm{~km}$, and a strong global asymmetry stemming from earlier SASI activity remains imprinted on to the post-shock flow. Asymmetric accretion on to the PNS still continues, but the accretion rate is reduced by a factor of $\sim 2$ compared to model s20.

(iv) s27: our most massive model is based on the $27 \mathrm{M}_{\odot}$ solarmetallicity progenitor of Woosley et al. (2002) and has been discussed in greater detail in Hanke et al. (2013) and, for SASI-induced neutrino emission variations, by Tamborra et al. (2013, 2014a). Shock revival did not occur by the end of the simulation $575 \mathrm{~ms}$ after bounce. There are two episodes of pronounced SASI activity that are interrupted by a phase of transient shock expansion following the infall of the $\mathrm{Si} / \mathrm{O}$ interface. The first SASI phase takes place between 120 and $260 \mathrm{~ms}$ post-bounce and the second period sets in around $410 \mathrm{~ms}$ post-bounce and lasts until the end of the simulation.

\subsubsection{D models}

In addition to the $3 \mathrm{D}$ models, we also analyse two $2 \mathrm{D}$ models based on the same progenitor as s27.

(i) s27-2D: model s27-2D was simulated with the same numerical setup as s27 (see Hanke et al. 2013), with an initial grid resolution 
of $400 \times 88$ zones in $r$ and $\theta$ and the innermost $10 \mathrm{~km}$ being simulated in spherical symmetry to allow for optimal comparison with the 3D model. SASI activity sets in about $150 \mathrm{~ms}$ after core bounce. Between 220 and $240 \mathrm{~ms}$ after bounce the accretion rate drops significantly after the $\mathrm{Si} / \mathrm{O}$ shell interface has crossed the shock. The decreasing accretion rate leads to shock expansion, and shock revival occurs around $300 \mathrm{~ms}$ post-bounce.

(ii) G27-2D: in order to compare our results to those of a relativistic 2D simulation of the SASI-dominated s27 model, we also reanalyse the 2D model G27-2D presented by Müller et al. (2013), which was simulated with COCONUT-VERTEX (Müller, Janka \& Dimmelmeier 2010). Coconut (Dimmelmeier, Font \& Müller 2002; Dimmelmeier et al. 2005) uses a directionally unsplit implementation of the piecewise parabolic method (with an approximate Riemann solver) for general relativistic hydrodynamics in spherical polar coordinates. The metric equations are solved in the extended conformal flatness approximation (Cordero-Carrión et al. 2009). The model was simulated with an initial grid resolution of $400 \times 128$ zones in $r$ and $\theta$, with the innermost $1.6 \mathrm{~km}$ being simulated in spherical symmetry to reduce time-step limitations. For consistency, we recompute the GW amplitudes for this model based on the relativistic stress formula (appendix A of Müller et al. 2013) instead of the time-integrated quadrupole formula with centred differences as originally used by Müller et al. (2013). The stress formula leads to somewhat larger amplitudes particularly at late times when central differencing is no longer fully adequate due to the increasing signal frequency.

The model is characterized by strong post-shock convection for the first $50 \mathrm{~ms}$ after core bounce, which is followed by a phase of strong SASI activity. Around $120 \mathrm{~ms}$ after core bounce the average shock radius starts to increase steadily. The criterion for runaway shock expansion is met approximately $180 \mathrm{~ms}$ after bounce and the shock is successfully revived at $\sim 210 \mathrm{~ms}$ post bounce.

The evolution of models G27-2D and s27-2D differs significantly during the pre-explosion phase: in G27-2D large-scale deformation of the shock already occurs $\sim 50 \mathrm{~ms}$ after bounce, without a preceding phase of hot-bubble convection (Müller et al. 2013). s272D develops SASI activity later: since the average shock radius is $\sim 20-30 \mathrm{~km}$ larger in model s27-2D than in model G27-2D, conditions favour the development of neutrino-driven convection. Consequently, s27-2D shows an initial phase of convection before SASI activity sets in when the shock starts to retract $\sim 100-150 \mathrm{~ms}$ after bounce. Due to the early development of SASI activity in model G27-2D at a time when the accretion rate is high, particularly strong supersonic downflows on to the PNS develop.

A possible reason for stronger and earlier SASI activity in model G27-2D is that, in contrast to model s27-2D, model G27-2D exhibits a phase of strong prompt post-shock convection (between a few ms after bounce and about $50 \mathrm{~ms}$ after bounce), which leaves the shock appreciably deformed with $\left|a_{1}\right| / a_{0} \sim 0.01-0.02$ as shown in fig. 7 of Müller, Janka \& Heger (2012b). Therefore, the SASI amplitude only needs to grow by a factor of $\sim 30$ to reach the non-linear regime. In Hanke et al. (2013, fig. 2), the $l=1$ amplitude is much smaller at early times. Such differences in the post-bounce evolution can have a variety of reasons. Besides the pure stochasticity of simulations, the initial perturbations may also play a role: model G27-2D was simulated in 2D from the onset of core collapse, while model s27-2D was started from a spherical model with seed perturbations imposed $15 \mathrm{~ms}$ after core bounce. The presence or absence of strong prompt post-shock convection also depends on the details of the entropy and electron fraction profiles, which are determined by the exact shock dynamics during the first milliseconds after core bounce.
Without a very careful analysis of all the differences between the two simulations, we are not able to localize the origin of the differences between model G27-2D and model s27-2D in the different gravity treatment or any of the other aforementioned aspects.

Despite (or because of) the differences in the dynamics of the postshock flow to s27-2D, model G27-2D is useful for illustrating the differences of the 3D model to the extreme end of the spectrum of recent 2D models in terms of peak GW amplitude and illustrates the mechanism of GW emission by stochastic surface g-mode excitation due to overshooting plumes from the gain region (Marek et al. 2009; Murphy et al. 2009; Müller et al. 2013) in the clearest form.

\section{STRUCTURE AND ORIGIN OF THE GRAVITATIONAL WAVE SIGNAL}

The different 3D models used in our analysis probe distinctly different regimes that can be encountered in supernova cores. In this section, we will investigate how these dynamical differences are reflected in the GW signals. We also compare our 3D models to the two 2D models and investigate how and why the GW signal changes when going from $2 \mathrm{D}$ to $3 \mathrm{D}$.

\subsection{Gravitational wave extraction}

In order to extract the GW signal from the hydrodynamical simulations, we post-process our simulations using the quadrupole stress formula (Finn 1989; Nakamura \& Oohara 1989; Blanchet, Damour $\&$ Schaefer 1990). Here, we only give a concise description of the formalism and refer the reader to Müller et al. (2012a) ${ }^{2}$ for a full explanation.

In the transverse traceless (TT) gauge and the far-field limit, the metric perturbation, $\boldsymbol{h}^{\mathrm{TT}}$, can be expressed in terms of the amplitudes of the two independent polarization modes in the following way,

$\boldsymbol{h}^{\mathrm{TT}}(\boldsymbol{X}, t)=\frac{1}{D}\left[A_{+} \boldsymbol{e}_{+}+A_{\times} \boldsymbol{e}_{\times}\right]$.

Here, $D$ denotes the distance between the source and the observer, $A_{+}$denotes the wave amplitude of the plus-polarized mode, $A_{\times}$is the wave amplitude of the cross-polarized mode and $\boldsymbol{e}_{\times}$and $\boldsymbol{e}_{+}$ denote the unit polarization tensors. The unit polarization tensors are given by

$\boldsymbol{e}_{+}=\boldsymbol{e}_{\theta} \otimes \boldsymbol{e}_{\theta}-\boldsymbol{e}_{\phi} \otimes \boldsymbol{e}_{\phi}$

$\boldsymbol{e}_{\mathrm{x}}=\boldsymbol{e}_{\theta} \otimes \boldsymbol{e}_{\phi}+\boldsymbol{e}_{\phi} \otimes \boldsymbol{e}_{\theta}$,

where $\boldsymbol{e}_{\theta}$ and $\boldsymbol{e}_{\phi}$ are the unit vectors in the $\theta$ and $\phi$ direction of a spherical coordinate system and $\otimes$ denotes the tensor product. Using the quadrupole approximation in the slow-motion limit, the amplitudes $A_{\times}$and $A_{+}$can be computed from the second time derivative of the symmetric trace-free (STF) part of the mass quadrupole tensor $Q$ (Oohara, Nakamura \& Shibata 1997),

$A_{+}=\ddot{Q}_{\theta \theta}-\ddot{Q}_{\phi \phi}$,

$A_{\times}=2 \ddot{Q}_{\theta \phi}$.

The components of $Q$ in the orthonormal basis associated with spherical polar coordinates used in this formula can be obtained

\footnotetext{
${ }^{2}$ Note, however, that the description of the formalism in Müller et al. (2012a) contains some typos: their equation 24 is incomplete. The superscript TT is missing from $\ddot{Q}_{i j}$, as is also the case in equations (22) and (23), and, more importantly, the trace term is missing.
} 
from the Cartesian components $\ddot{Q}_{i j}$ of $\ddot{Q}$ (Nakamura, Oohara \& Kojima 1987; Oohara et al. 1997). Using the continuity and momentum equations to eliminate time derivatives (Finn 1989; Blanchet et al. 1990; Oohara et al. 1997), the Cartesian components can be obtained as

$\ddot{Q}_{i j}=\operatorname{STF}\left[2 \frac{G}{c^{4}} \int \mathrm{d}^{3} x \rho\left(v_{i} v_{j}-x_{i} \partial_{j} \Phi\right)\right]$.

Here, $G$ is Newton's gravitational constant, $c$ is the speed of light, and $v_{i}$ and $x_{i}$ are the Cartesian velocity components and coordinates $(i=1,2,3)$, respectively. The gravitational potential $\Phi$ is the gravitational potential used in the simulations (with post-Newtonian corrections taken into account). STF denotes the projection operator on to the STF part. The advantage of this form is that the second-order time derivatives are transformed into first-order spatial derivatives, thus circumventing problems associated with the numerical evaluation of second-order time derivatives. Using standard coordinate transformations between Cartesian and spherical coordinates, we obtain (Nakamura et al. 1987; Oohara et al. 1997) the components $\ddot{Q}_{\theta \theta}, \ddot{Q}_{\phi \phi}$, and $\ddot{Q}_{\theta \phi}$ needed in equations (4) and (5),

$$
\begin{aligned}
\ddot{Q}_{\theta \phi}= & \left(\ddot{Q}_{22}-\ddot{Q}_{11}\right) \cos \theta \sin \phi \cos \phi \\
& +\ddot{Q}_{12} \cos \theta\left(\cos ^{2} \phi-\sin ^{2} \phi\right) \\
& +\ddot{Q}_{13} \sin \theta \sin \phi-\ddot{Q}_{23} \sin \theta \cos \phi,
\end{aligned}
$$

$\ddot{Q}_{\phi \phi}=\ddot{Q}_{11} \sin ^{2} \phi+\ddot{Q}_{22} \cos ^{2} \phi-2 \ddot{Q}_{12} \sin \phi \cos \phi$

and

$$
\begin{aligned}
\ddot{Q}_{\theta \theta}= & \left(\ddot{Q}_{11} \cos ^{2} \phi+\ddot{Q}_{22} \sin ^{2} \phi+2 \ddot{Q}_{12} \sin \phi \cos \phi\right) \cos ^{2} \theta \\
& +\ddot{Q}_{33} \sin ^{2} \theta-2\left(\ddot{Q}_{13} \cos \phi+\ddot{Q}_{23} \sin \phi\right) \sin \theta \cos \theta .
\end{aligned}
$$

In axisymmetry the only independent component of $\boldsymbol{h}^{\mathrm{TT}}$ is

$h_{\theta \theta}^{\mathrm{TT}}=\frac{1}{8} \sqrt{\frac{15}{\pi}} \sin ^{2} \theta \frac{A_{20}^{\mathrm{E} 2}}{D}$,

where $D$ is the distance to the source, $\theta$ is the inclination angle of the observer with respect to the axis of symmetry and $A_{20}^{\mathrm{E} 2}$ represents the only non-zero quadrupole amplitude. In spherical coordinates, $A_{20}^{\mathrm{E} 2}$ can be expressed as follows:

$$
\begin{aligned}
A_{20}^{\mathrm{E} 2}(t)= & \frac{G}{c^{4}} \frac{16 \pi^{3 / 2}}{\sqrt{15}} \int_{-1}^{1} \int_{0}^{\infty} \rho\left[v_{r}^{2}\left(3 z^{2}-1\right)\right. \\
& +v_{\theta}^{2}\left(2-3 z^{2}\right)-v_{\phi}^{2}-6 v_{\mathrm{r}} v_{\theta} z \sqrt{1-z^{2}} \\
& \left.+r \partial_{r} \Phi\left(3 z^{2}-1\right)+3 \partial_{\theta} z \sqrt{1-z^{2}} \Phi\right] r^{2} \mathrm{~d} r \mathrm{~d} z .
\end{aligned}
$$

Here, $v_{i}$ and $\partial_{i}(i=r, \theta, \phi)$ represent the velocity components and derivatives, respectively, along the basis vectors of the spherical coordinate system, and $z \equiv \cos \theta$. For details, we refer the reader to Müller \& Janka (1997).

In this work, we disregard the contribution of anisotropic neutrino emission (Epstein 1978) to the GW signal. Due to its low-frequency nature, it is of minor relevance for the detectability and does not affect the waveforms appreciably in the frequency range $\gtrsim 50 \mathrm{~Hz}$ that is of primary interest to us in this work.

\subsection{Overview of waveforms}

\subsubsection{Waveforms}

Amplitudes for GWs generated by asymmetric mass motions are shown in Fig. 1. For each progenitor, we show two panels representing the cross and plus polarization for two different observer positions. The two columns show the amplitudes for two different viewing angles, the right and left column representing observers situated along the $z$-axis (pole) and $x$-axis (equator) of the computational grid, respectively. ${ }^{3}$ Since our (non-rotating) models do not exhibit a signal from a rotational bounce, and since (EoS-dependent) prompt post-shock convection is weak, the waveforms exhibit an initial quiescent phase. This is followed by a rather stochastic phase with amplitudes of several centimetres once convection or the SASI have fully developed. The correlation of stronger $\mathrm{GW}$ emission with the onset of strong, non-linear SASI activity in model s20, s20s and s27 is illustrated by dashed and solid lines bracketing phases of particularly violent SASI oscillations.

The signal from early SASI activity triggered by prompt convection a few tens of milliseconds after bounce, which is typically rather prominent in 2D (Murphy \& Burrows 2008a; Marek et al. 2009; Yakunin et al. 2010, 2015; Müller et al. 2013), is thus strongly reduced in 3D. It is only clearly visible in the waveforms of s20 and s20s, while s11.2 and s27 only show traces of this component in some directions. The stochastic modulation of the later signal is reminiscent of 2D models, but the amplitudes are significantly lower $(\lesssim 4 \mathrm{~cm})$ compared to several tens of cm in first-principle 2D models (Marek et al. 2009; Yakunin et al. 2010; Müller et al. 2013; Yakunin et al. 2015). The reduction in $3 \mathrm{D}$ is far stronger than could be expected from a mere projection effect (in agreement with parametrized models of Müller et al. 2012a).

Prior to a post-bounce time of $\sim 200 \mathrm{~ms}$, the waveforms for the three SASI-dominated models are clearly dominated by a lowfrequency signal (in very much the same fashion as for early SASI activity a few tens of milliseconds after bounce in 2D). This already indicates that the relative importance of the low- and highfrequency components of the signal during the accretion phase is different in 3D compared to 2D, where low-frequency emission (triggered by prompt convection) only dominates for the first tens of milliseconds.

In the exploding model s20s with $g_{\mathrm{a}}^{\mathrm{s}}=-0.2$, we observe a tendency towards somewhat higher peak amplitudes than during the accretion phase well after the onset of the explosion $(\sim 300 \mathrm{~ms})$. This tendency is, however, much less pronounced than in $2 \mathrm{D}$ models. The monotonous 'tail' in the matter signal from anisotropic shock expansion (Murphy et al. 2009; Yakunin et al. 2010) is noticeably absent, although no undue importance should be attached to this because it may take several hundreds of milliseconds for the tail to develop (Müller et al. 2013).

\subsubsection{Energy spectra}

Time-integrated energy spectra for each of the models are shown in Fig. 2. These are computed from the Cartesian components of the mass quadrupole tensor as

$$
\begin{aligned}
\frac{\mathrm{d} E}{\mathrm{~d} f}= & \frac{2 c^{3}}{5 G}(2 \pi f)^{2}\left[\left|\widetilde{\ddot{Q}}_{x x}\right|^{2}+\left|\widetilde{\ddot{Q}}_{y y}\right|^{2}+\left|\widetilde{\ddot{Q}}_{z z}\right|^{2}\right. \\
& \left.+2\left(\left|\widetilde{\ddot{Q}}_{x y}\right|^{2}+\left|\widetilde{\ddot{Q}}_{x z}\right|^{2}+\left|\widetilde{\ddot{Q}}_{y z}\right|^{2}\right)\right],
\end{aligned}
$$

\footnotetext{
${ }^{3}$ In our post-processing, we chose to sample the GW signal at observer directions corresponding to cell centres of the simulation grid and as a consequence the two directions do not exactly correspond to the north pole $(\theta=0, \phi=0)$ and the equator $(\theta=\pi, \phi=0)$, but are offset by half of the angular resolution. Hence, the coordinates of the polar and equatorial observer become $(\pi / 176, \pi / 176)$ and $(\pi-\pi / 176, \pi / 176)$, respectively.
} 


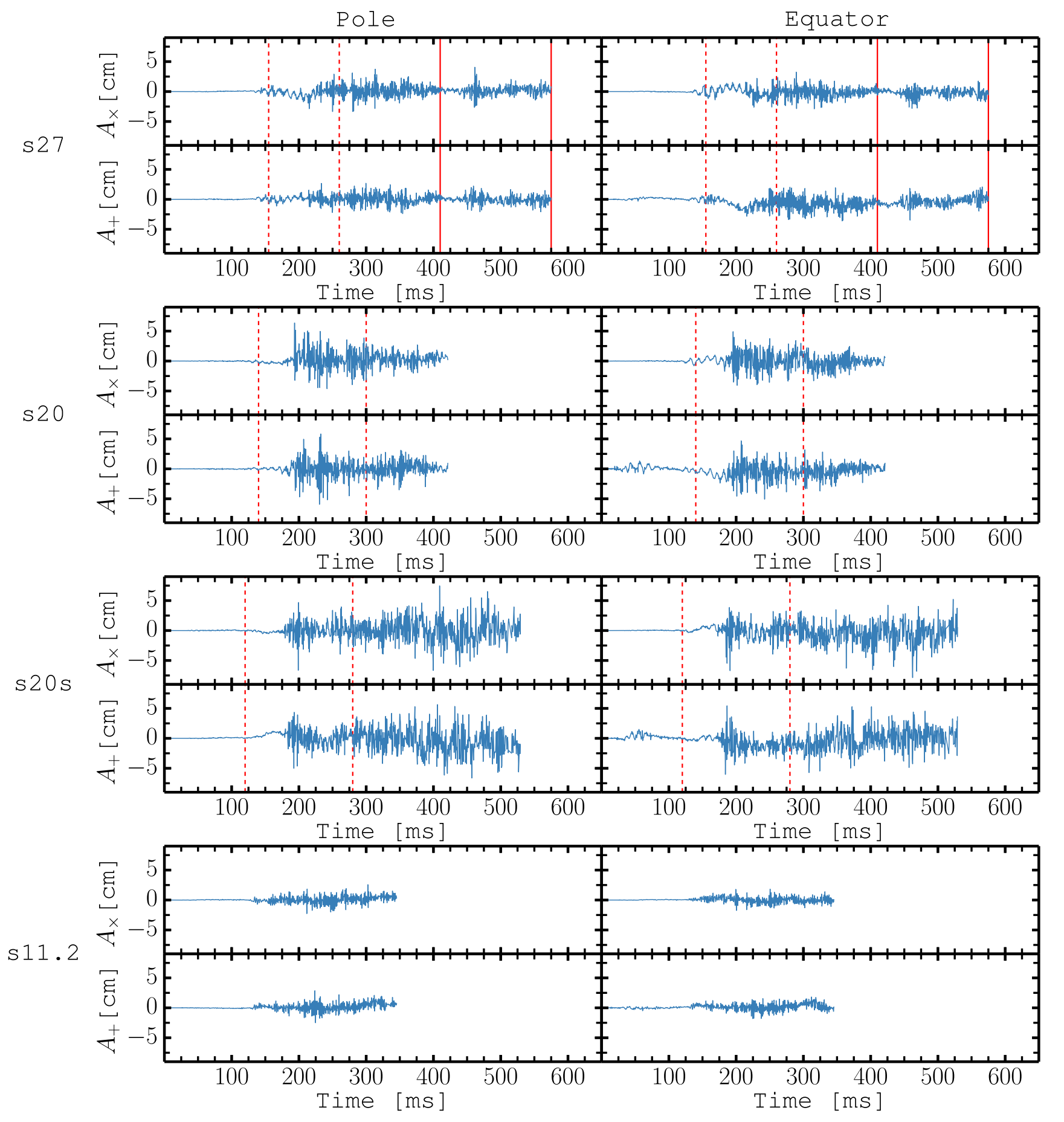

Figure 1. GW amplitudes $A_{+}$and $A_{\times}$as functions of time after core bounce. From the top: s27, s20, s20s and s11, respectively. The two columns show the amplitudes for two different viewing angles: an observer situated along the $z$-axis (pole; left) and an other observer along the $x$-axis (equator; right) of the computational grid, respectively. Episodes of strong SASI activity occur between the vertical red lines; dashed and solid lines are used for model s27 to distinguish between two different SASI episodes.

where tildes denote Fourier transforms, and $f$ is the frequency. We define the Fourier transform as follows:

$\widetilde{\ddot{Q}}_{i j}(f)=\int_{-\infty}^{\infty} \ddot{Q}_{i j}(t) e^{-2 \pi i f t} \mathrm{~d} t$.

The energy spectra of the three SASI-dominated models are relatively flat. This is significantly different from 2D models, where the energy spectra are dominated by a peak at several hundreds of Hz (Marek et al. 2009; Müller et al. 2013; Yakunin et al. 2015). Model s11.2, on the other hand, more closely resembles the 2D energy spectra, although the total energy emitted in GWs is considerably lower than in typical $2 \mathrm{D}$ models. In addition, the peak values of $\mathrm{d} E / \mathrm{d} f$ are considerably higher in the SASI models than for $\mathrm{s} 11.2$. 
s27

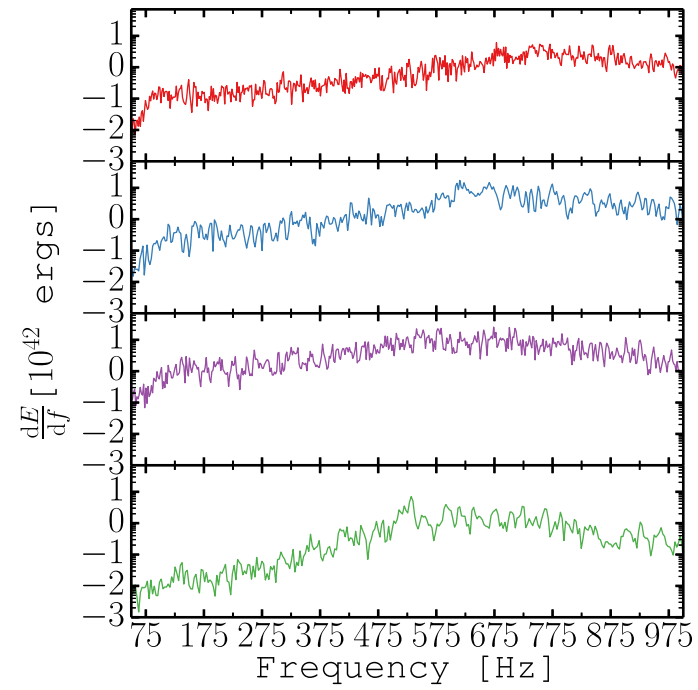

Figure 2. Time-integrated GW energy spectra $\mathrm{d} E / \mathrm{d} f$ for models s27, s20, s11.2 and s20s (top to bottom). The spectra are computed from the Fourier transform of the entire waveform without applying a window function. The $y$-axis is given in a logarithmic scale.

\subsubsection{The signal in the time-frequency domain}

In order to dissect the signal further, we apply a short-time Fourier transform (STFT) to our waveforms. For a discrete time series, the STFT is obtained by applying the discrete Fourier transform (DFT) to the signal with a sliding window. In this work, we define the DFT, $\widetilde{X}_{k}$, as follows:

$\widetilde{X}_{k}\left(f_{k}\right)=\frac{1}{M} \sum_{m=1}^{M} x_{m} \mathrm{e}^{-2 \pi \mathrm{i} k m / N}$,

Here, $x_{m}$ is the time series obtained by sampling the underlying continuous signal at $M$ discrete times. $f_{k}=k / T$ is the frequency of bin $k$, where $T$ is the duration of the signal.

The resulting amplitude spectrograms for a sliding window of $50 \mathrm{~ms}$ are shown in Fig. 3. The spectrograms show the sum of the squared Fourier components of the cross and plus polarization modes, $\left|\widetilde{A_{+}}\right|^{2}+\left|\widetilde{A_{\times}}\right|^{2}$. Before applying the DFT, we convolve the signal with a Kaiser window with shape parameter $\beta=2.5$. Frequencies below $50 \mathrm{~Hz}$ and above $1100 \mathrm{~Hz}$ are filtered out of the resulting DFT. The amplitude spectrograms are computed for the same two observer directions as before.

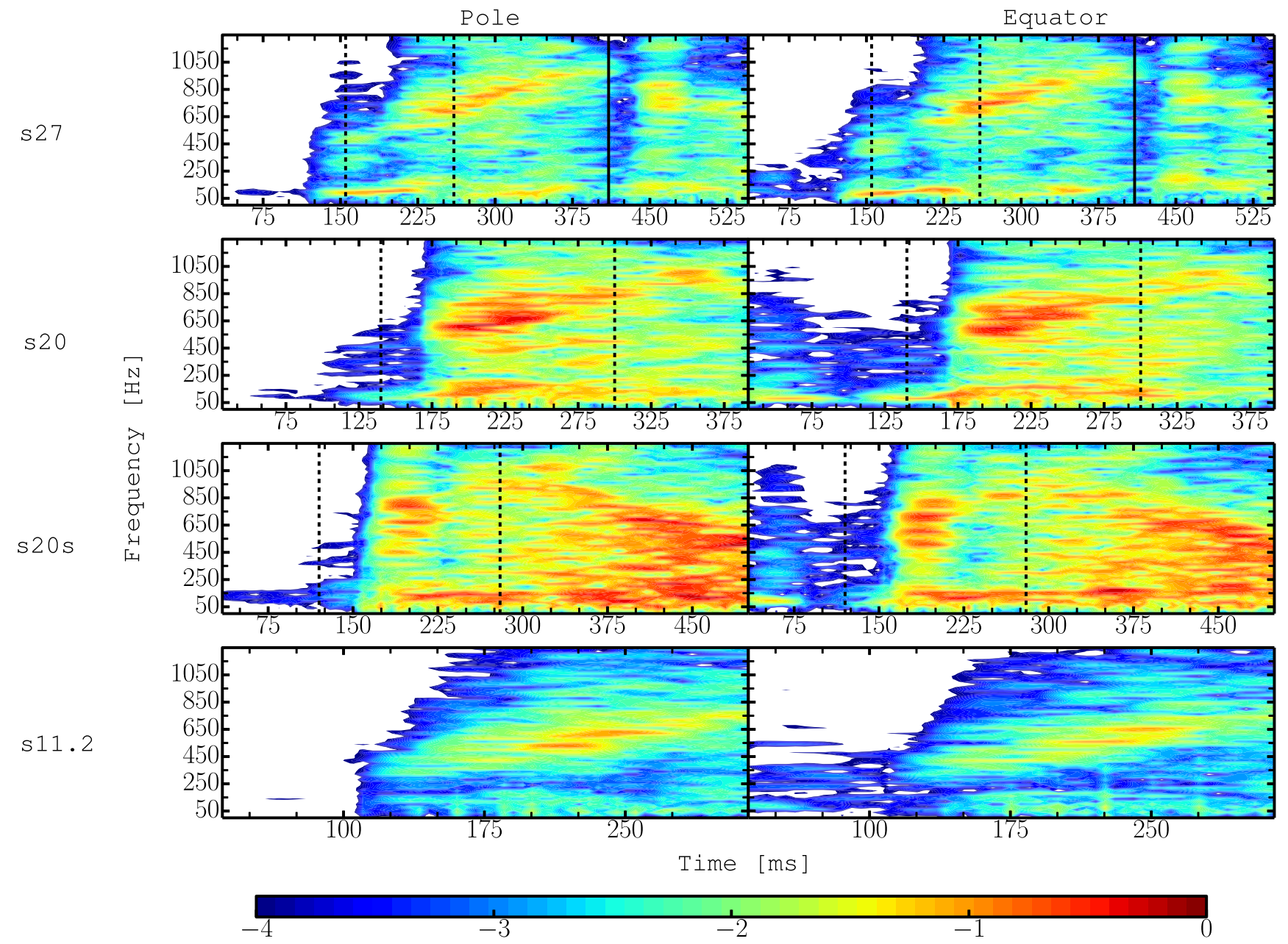

Figure 3. Amplitude spectrograms for a sliding window of $50 \mathrm{~ms}$ and two different observer directions, summed over the two polarization modes $\left(\left|\widetilde{A}_{+}\right|^{2}+\right.$ $\left|\widetilde{A}_{\times}\right|^{2}$ ). The different rows show the results for models s27, s20, s20s and s11.2. (top to bottom). The two columns show the spectrograms for two different viewing angles, the right and the left column represent observers situated along the $z$-axis (pole) and $x$-axis (equator) of the computational grid, respectively. The time is given in ms after core bounce. Vertical lines bracket SASI episodes. All panels have been normalized by the same global factor. The colour bar is given in a logarithmic scale. 
All of the models exhibit the distinct high-frequency (here defined to be emission at frequencies greater than $250 \mathrm{~Hz}$ ) component familiar from 2D models with a slow, secular increase in the peak frequency.

The SASI-dominated models stand apart from model s11.2 in that they show an additional low-frequency component (below $250 \mathrm{~Hz}$ ) at late times (i.e. not associated with prompt convection). No such distinct low-frequency emission has been observed in spectrograms from 2D models (Murphy et al. 2009; Müller et al. 2013). The lowfrequency component is clearly separated from the high-frequency emission by a 'quiet zone' in the spectrograms. The frequency structure of the low-frequency component is rather complicated, and especially for models s20 and s20s it is rather broad-banded. There is also a directional dependence as can be seen, for example, from the later onset of low-frequency emission in the polar direction compared to the equatorial direction, in model s20 (second row in Fig. 3).

During the explosion phase, we find increased power in the highfrequency band corresponding to the increased peak amplitudes discussed in Section 3.2.1. However, the most conspicuous change after the onset of the explosion consists in a considerable increase of broad-band power at low frequencies. Close inspection of Fig. 1 shows that the enhanced low-frequency emission can also be seen directly in the amplitudes: the amplitude 'band' defined by stochastic high-frequency oscillations is clearly not centred at zero amplitude, but exhibits a significant low-frequency modulation.

Typical frequencies of the order of $100 \mathrm{~Hz}$ as well as a vague temporal correlation of the low-frequency emission with periods of strong sloshing/spiral motions suggest a connection with SASI activity. However, model s27 (top row in Fig. 3) also shows lowfrequency emission during the phase between 280 and $350 \mathrm{~ms}$ after bounce when the SASI is relatively quiet. If the signal were directly due to the SASI, one would expect the phases of strong SASI and strong low-frequency emission to coincide. There may also be correlations between the low- and the high-frequency emission as suggested by the fact that model s 20 with the strongest low-frequency emission also exhibits the strongest high-frequency signal. Moreover, the source of enhanced low-frequency emission after shock revival is not immediately intuitive since the SASI no longer operates during this phase. This calls for a closer investigation of the hydrodynamic processes responsible for the emission of the two signal components.

\subsection{Spatial location of underlying hydrodynamical instabilities}

Which regions of the simulation volume contribute to the different GW components? The emission of GWs cannot be strictly localized, but one can none the less still partition the computational volume in the quadrupole formula (6) into different regions, and consider the formal contributions of each of these to the total signal. While this may not amount to a strict localization of GW emission as coming from a specific region, such a partitioning nevertheless helps to detect fluid motions with the required temporal and frequency structure to account for different components of the signal in conjunction with the temporal evolution of the amplitudes and the spectral power. This procedure cannot replace a more rigorous identification of GW-emitting modes, which must, however, be left to the future.

In this work, we divide the integration volume into three layers A, $\mathrm{B}$ and C (see Fig. 4). The PNS is split into two layers, the "convective layer' (layer A) and the convectively stable 'surface layer'(layer B). The convectively stable inner core $(r<10 \mathrm{~km})$ is not considered

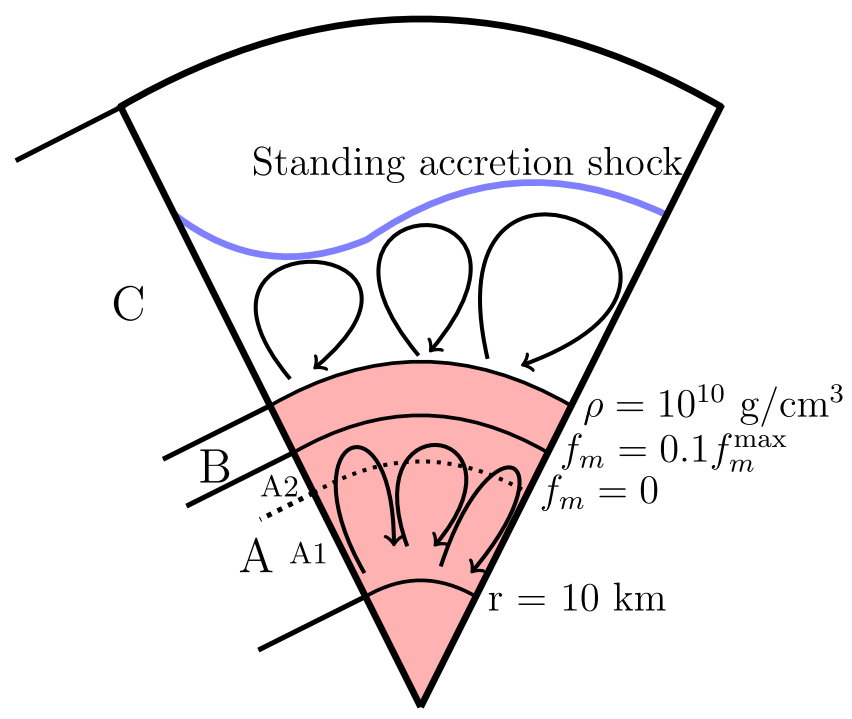

Figure 4. Schematic overview of the regions of hydrodynamical activity. In Section 3.3, we investigate the contribution to the total GW signal from three different layers. The PNS, indicated by the shaded red area, is divided into two layers: layer A includes the convectively unstable region in the PNS (layer A1) and the overshooting layer A2 directly above it. The boundary between the convective layer and the overshooting layer is indicated by a dashed curve within layer A. The second layer, layer B, extends from the top of the overshooting region and out to the PNS surface, defined by a fiducial density of $10^{10} \mathrm{~g} \mathrm{~cm}^{-3}$. Layer $\mathrm{C}$ extends from the PNS surface to the outer boundary of our simulation volume. Layer $\mathrm{C}$ therefore includes the post-shock region, the standing accretion shock (indicated by the blue line) and the pre-shock region. Formal definitions of the boundaries between layers are given on the right-hand side, see Section 3.3 for details.

in our analysis because it is simulated in spherical symmetry and consequently does not contribute to the GW emission. A third layer (layer C) comprises the region between the outer boundary of the PNS (defined by a density of $10^{10} \mathrm{~g} \mathrm{~cm}^{-3}$ ) and the outer boundary of the grid. We refer to this region as 'post-shock' region because only motions in the post-shock region and the deceleration of matter at the shock effectively contribute to the signal from this layer.

The boundary between layer A and layer B is defined based on a horizontal averaging scheme from the stellar convection literature, see e.g. Nordlund, Stein \& Asplund (2009) and Viallet et al. (2013). We define volume-weighted horizontal averages (denoted by angled brackets) of any quantity $X$ such as velocity, density or pressure as follows,

$$
\langle X\rangle=\frac{\int X \mathrm{~d} \Omega}{\int \mathrm{d} \Omega} .
$$

The quantity $X$ is then decomposed into a mean and a fluctuating component,

$X=\langle X\rangle+X^{\prime}$.

For defining the boundary between the convective region and the stable surface region, we consider the turbulent mass flux $f_{\mathrm{m}}$,

$f_{\mathrm{m}}=\left\langle\rho^{\prime} v_{r}^{\prime}\right\rangle$.

Inside the convective region, heavier fluid is advected downwards while fluid that is lighter than average rises upwards. The turbulent mass flux will therefore always be negative in the convective layer. In the overshooting layer outside the PNS convection zone the situation is reversed, and $f_{\mathrm{m}}$ is positive as the overshooting, outward-moving plumes are denser than their surroundings. In our calculations, we 


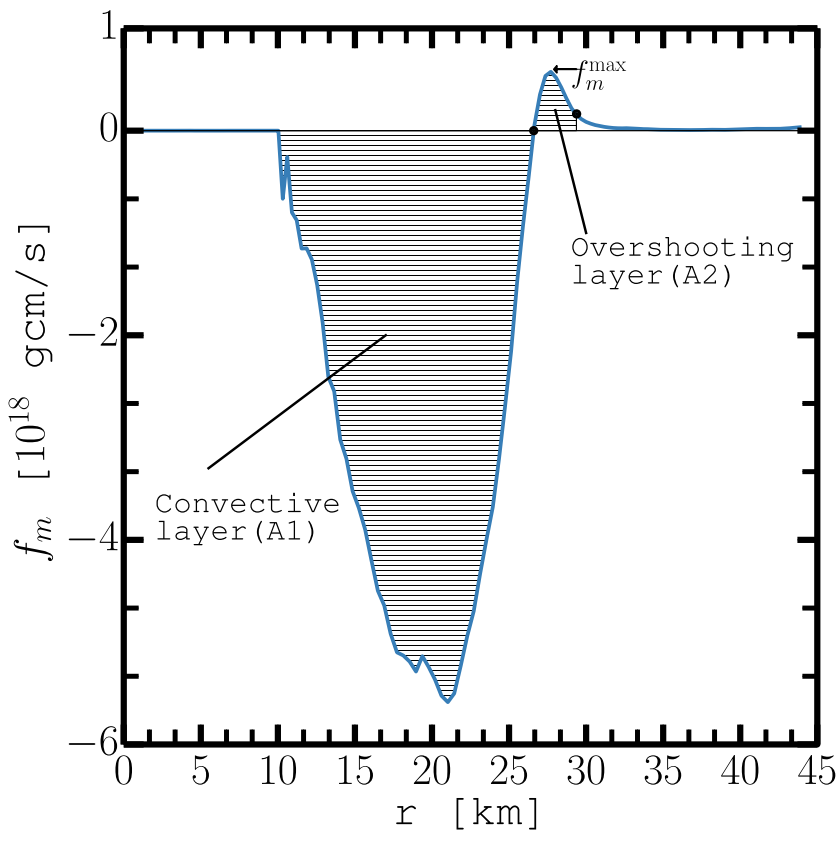

Figure 5. Turbulent mass flux $f_{\mathrm{m}}$ (blue curve) for model s27, calculated $192 \mathrm{~ms}$ after core bounce. The shaded region indicates the convectively unstable region and the overshooting layer, which are lumped together as layer A (see Fig. 4).
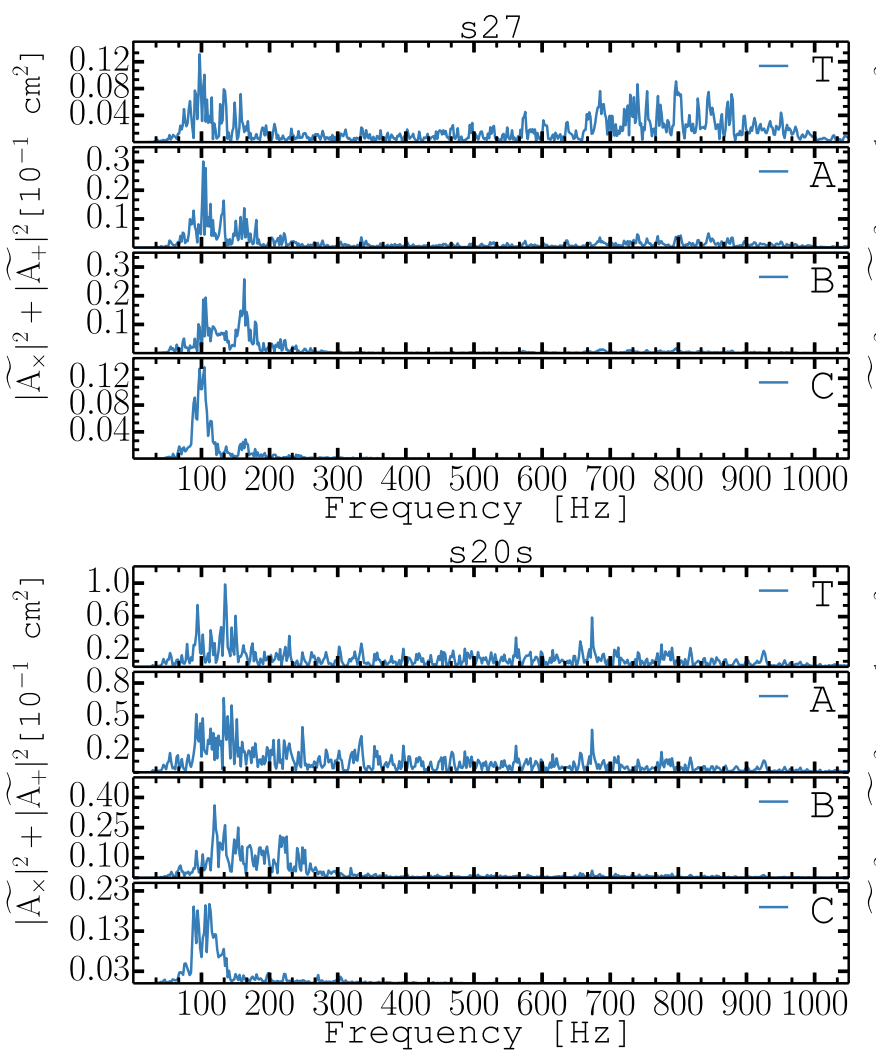

include the overshooting region in layer A. To capture properly both the convective zone and the region of overshooting, we define the boundary between layer A and layer B as the radius where

$f_{\mathrm{m}}=\left.0.1 f_{\mathrm{m}}^{\max }\right|_{r>r_{\max }}$,

where $f_{\mathrm{m}}^{\max }$ and $r_{\max }$ are the maximum value of the turbulent mass flux and the radius at which we find $f_{\mathrm{m}}^{\max }$, respectively. This definition can be more easily understood with the help of a radial profile of $f_{\mathrm{m}}$ as shown in Fig. 5 for model s27 at a post-bounce time of $192 \mathrm{~ms}$. Where necessary, we further distinguish between the convective layer (layer A1) and the overshooting layer (layer A2), which are separated by the radius where $f_{\mathrm{m}}=0$.

In Fig. 6, we plot the Fourier amplitudes of the GW amplitudes arising from each individual layer; these amplitudes are calculated from the full-time signal and for an observer situated at the pole, corresponding to the left column in Fig. 3. This figure has to be analysed with some care. Since we plot the square of the Fourier coefficients one cannot add the values of layers A, B and C together and recover the value for the total signal. In addition, artefacts can arise due to effects at the boundaries between layers, as in the case of model s20 (top right panel of Fig. 6). There is an artificially strong peak at $160 \mathrm{~Hz}$, particularly from layer B. We have confirmed that shifting the boundary between layers A and B inwards reduces this peak significantly. The exact values of the low-frequency amplitudes are sensitive to the boundary definition, but the fact that all three layers contribute to emission below $250 \mathrm{~Hz}$ is robust. The highfrequency component is less affected by such artefacts since the high-frequency emission is mostly confined to layer A.
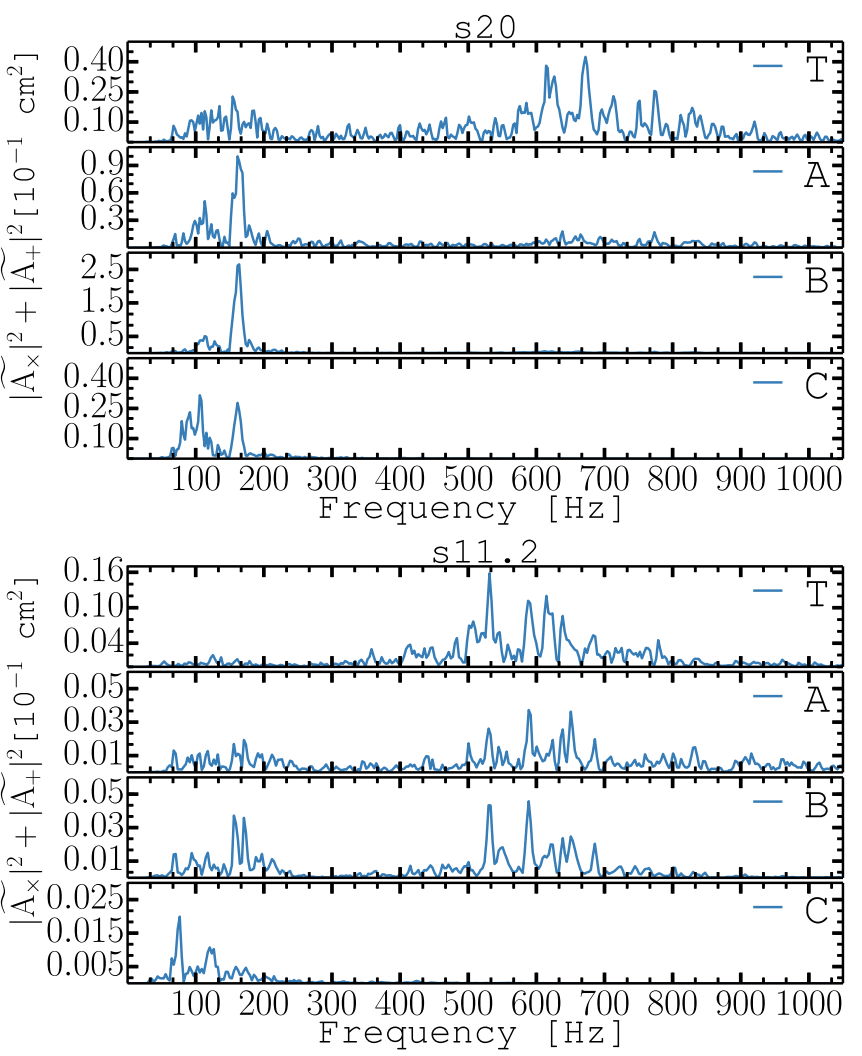

Figure 6. Squared Fourier amplitudes of the total volume-integrated GW signal and of the signal contributions arising from the three different layers of the simulation volume. From the top: total signal (T), the PNS convective region and the overshooting layer (A), the PNS surface layer (B), and the volume between the PNS surface and the outer grid boundary (C). Top row: the left and right columns show the results for models s27 and s20, respectively. Bottom row: the left and right columns show the results for models s20s and s11.2. See Fig. 4 for a sketch of the three regions used for this analysis. The Fourier amplitudes are calculated according to equations (14), (4) and (5). 
The results of this dissection of the contributions to the integral in equation (6) are somewhat unexpected. The high-frequency emission mostly stems from aspherical mass motions in layer A and there is only a minor contribution from layer $\mathrm{B}$, which has been posited as the crucial region for GW emission during the pre-explosion phase in works based on 2D simulations (Marek et al. 2009; Murphy et al. 2009; Müller et al. 2013). Aspherical mass motions in layer $\mathrm{C}$ hardly contribute to this component at all.

By contrast, all three regions contribute to the low-frequency signal (i.e. emission at frequencies lower than $250 \mathrm{~Hz}$ ) to a similar degree. This is also surprising if the dominant frequency of this component appears to be set by the SASI as speculated before. In this case, one might expect that the fluid motions responsible for $\mathrm{GW}$ emission are propagating waves in layer $\mathrm{C}$ and perhaps layer $\mathrm{B}$, where the conversion of vorticity perturbations into acoustic perturbations occurs in the SASI feedback cycle.

\subsection{Origin of high-frequency emission}

What do these findings imply about the physical mechanisms that give rise to $\mathrm{GW}$ emission and determine their frequency? Let us first address the high-frequency signal. Recent 2D studies have connected GW emission at $\gtrsim 500 \mathrm{~Hz}$ to oscillatory modes (g modes) excited either in the PNS surface (layer B) from above by downflows impinging on to the PNS (Marek et al. 2009; Murphy et al. 2009; Müller et al. 2013), or from below by PNS convection (Marek et al. 2009; Müller et al. 2012a, 2013). Prior to shock revival, the excitation of oscillations by mass motions in the gain layer was found to be dominant, with PNS convection taking over as the dominant excitation mechanism only after the onset of the explosion (Müller et al. 2012a, 2013). The typical angular frequency of such processes is roughly given by the Brunt-Väisälä frequency, $N$, in the convectively stable region between the gain region and the PNS convection zone,

$N^{2}=\frac{1}{\rho} \frac{\partial \Phi}{\partial r}\left[\frac{1}{c_{\mathrm{s}}^{2}} \frac{\partial P}{\partial r}-\frac{\partial \rho}{\partial r}\right]$

where $c_{\mathrm{S}}$ is the sound speed. Müller et al. (2013) further investigated the dependence of this frequency on the mass $M$, the radius $R$ and the surface temperature $T$ of the PNS to explain the secular increase of $N$ during the contraction of the PNS and a tendency towards higher frequencies for more massive neutron stars.

Our results confirm that the peak frequency of the high-frequency GW emission is still set by the Brunt-Väisälä frequency in 3D and therefore point to a similar role of buoyancy forces in determining the spectral structure of the high-frequency component. As shown in Fig. 7 for model s27, we find very good agreement between the peak GW frequency, $f_{\text {peak }}$, and the Brunt-Väisälä frequency, $N$, calculated at the outer boundary of the overshooting layer (the boundary between layers $\mathrm{A}$ and $\mathrm{B}$ ). Here, $f_{\text {peak }}$ denotes the frequency with the highest Fourier amplitude above $250 \mathrm{~Hz}$. Superficially, there appears to be a discrepancy at post-bounce times later than $400 \mathrm{~ms}$, where $f_{\text {peak }}$ seems to decrease again. This, however, is purely an artefact of the sampling rate of $0.5 \mathrm{~ms}$ in the simulations, which results in a Nyquist frequency of $1000 \mathrm{~Hz}$. The peak frequency is therefore aliased into the region below $1000 \mathrm{~Hz}$. If this is taken into account, there is in fact good agreement between the BruntVäisälä frequency of $\sim 1300 \mathrm{~Hz}$ and the aliased peak GW frequency of $\sim 700 \mathrm{~Hz}$ at the end of the simulation.

The dominant excitation mechanism for these oscillatory motions in layers A and B is, however, remarkably different from previous 2D models. While allowing for a minor contribution from PNS

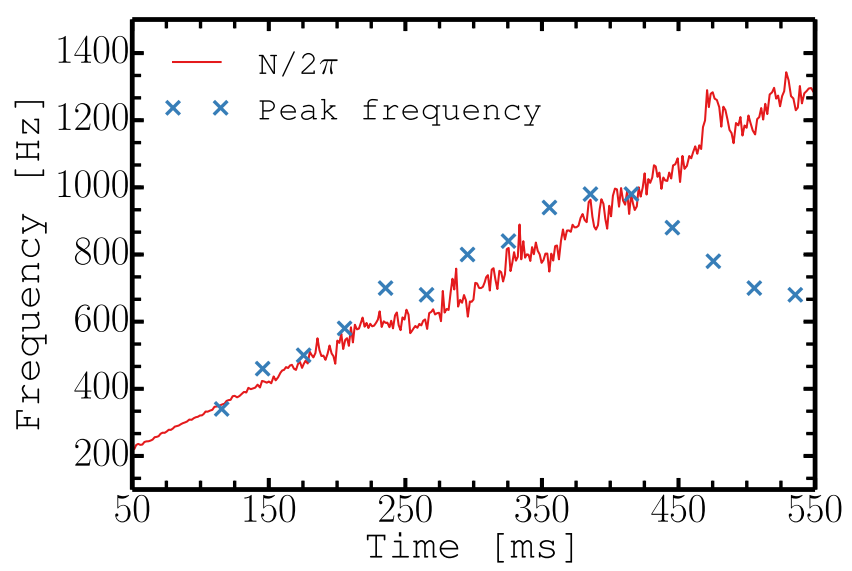

Figure 7. Frequency of strongest GW emission above $250 \mathrm{~Hz}$ in the spectrogram of model s 27 as a function of time (blue crosses). We also plot the expected characteristic frequency of GW emission excited by buoyancy effects in the PNS surface layer B and the overshooting layer A2 (red curve, see definition in equation 19). The exact value of $N$ depends on the radius where equation (19) is evaluated, but we find similar numerical values within layers B and A2. Note that the trends seen for model s 27 are common to all our models.

convection to the total signal during the pre-explosion phase, most of the signal in 2D is found to originate from oscillations in layer $\mathrm{B}$ that are excited by convective plumes and/or the downflows of the SASI (Marek et al. 2009; Murphy et al. 2009; Müller et al. 2013). In this case, one would expect that the excited oscillation modes have large amplitudes mostly in the surface layer and that this layer contributes significantly to the GW signal. This is not the case in 3D as shown by Fig. 6. The dominant contribution from layer A rather suggests that oscillatory modes are predominantly excited from below by aspherical mass motions in the PNS convection zone and are confined mostly to the overshooting layer, which acts as frequency stabilizer. This assessment is also compatible with the temporal evolution of the amplitudes and the power in the spectrograms. The amplitudes (Fig. 1) show modest temporal variations after an initial GW-quiet phase of 150-200 ms and little response to strong activity of SASI and convection, which argues against efficient excitation of surface $g$ modes by motions in the gain region. The spectrograms (Fig. 3) point to the same conclusion, e.g. high-frequency emission is practically absent during the first phase of SASI activity in s27.

To confirm the crucial role of layer A2, we excluded this region from our analysis and found a large reduction of the energy carried away by high-frequency GWs,

$E_{\mathrm{GW}} \sim \int_{250 \mathrm{~Hz}}^{1100 \mathrm{~Hz}} \frac{\mathrm{d} E}{\mathrm{~d} f} \mathrm{~d} f$.

For model s27, we find a reduction of the GW energy by roughly a factor of 2 when excluding the overshooting layer A2. It is remarkable that the deeper regions of the PNS convection zone (layer A1) none the less contribute to the high-frequency signal with similar frequencies: there is no apparent reason for a correlation between the convective overturn time $T_{\text {conv }}$ (which sets the natural frequency for GWs from the bulk of the PNS convection zone as $1 / T_{\text {conv }}$ ) and the Brunt-Väisälä frequency in the overlying stable region.

\subsection{Comparison of high-frequency emission in 2D and 3D}

To further illustrate the differences between previously published 2D waveforms and our 3D results, we re-analyse the $\mathrm{GW}$ signal 

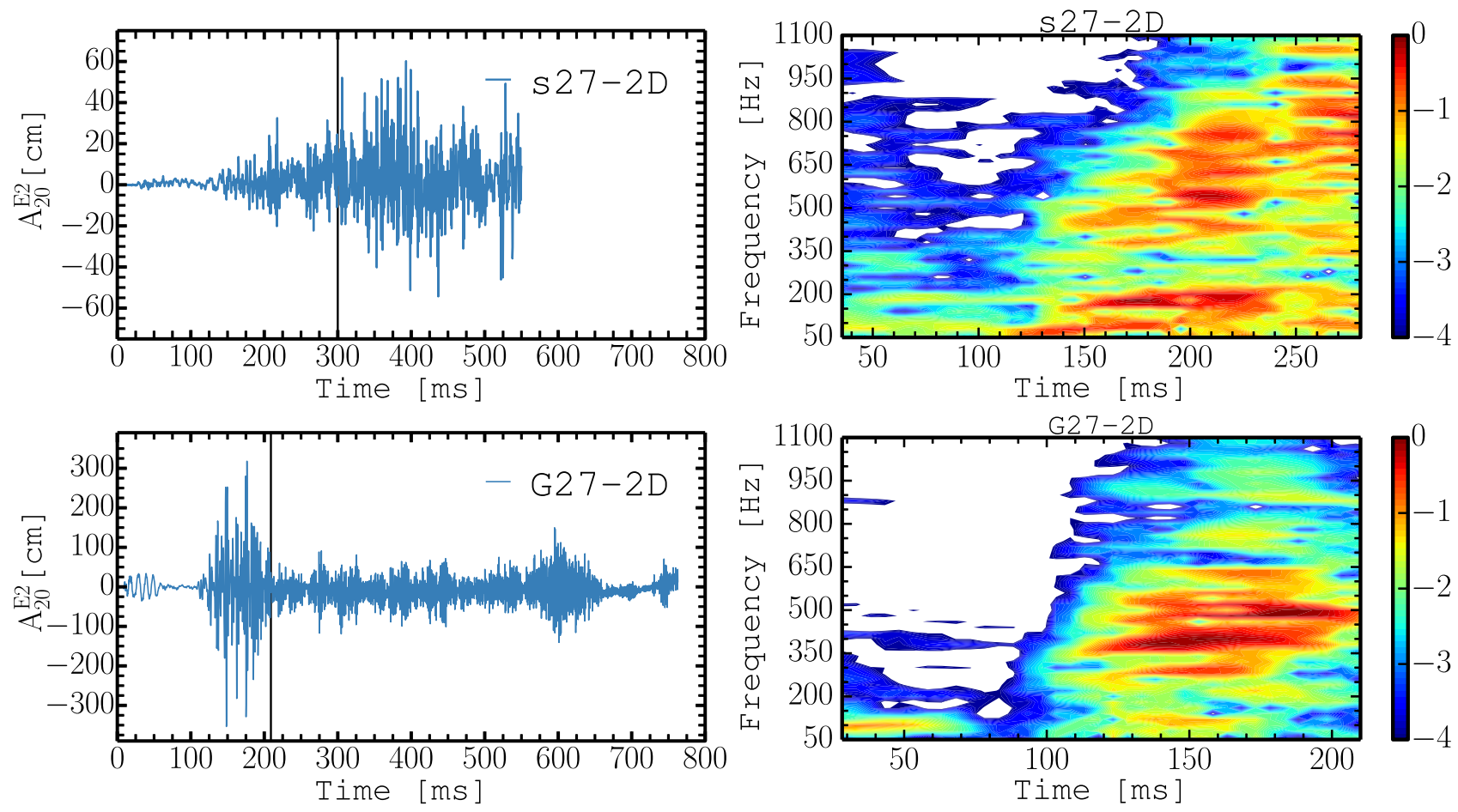

Figure 8. The GW amplitude, $\mathrm{A}_{E 2}^{20}$, as a function of time after bounce (left) and amplitude spectrograms in in logarithmic scale (right) for the two $2 \mathrm{D}$ models s27-2D (top row) and G27-2D (bottom row). For a useful comparison with the corresponding non-exploding 3D model, we only show the spectrograms for the time between bounce and the onset of the explosion.
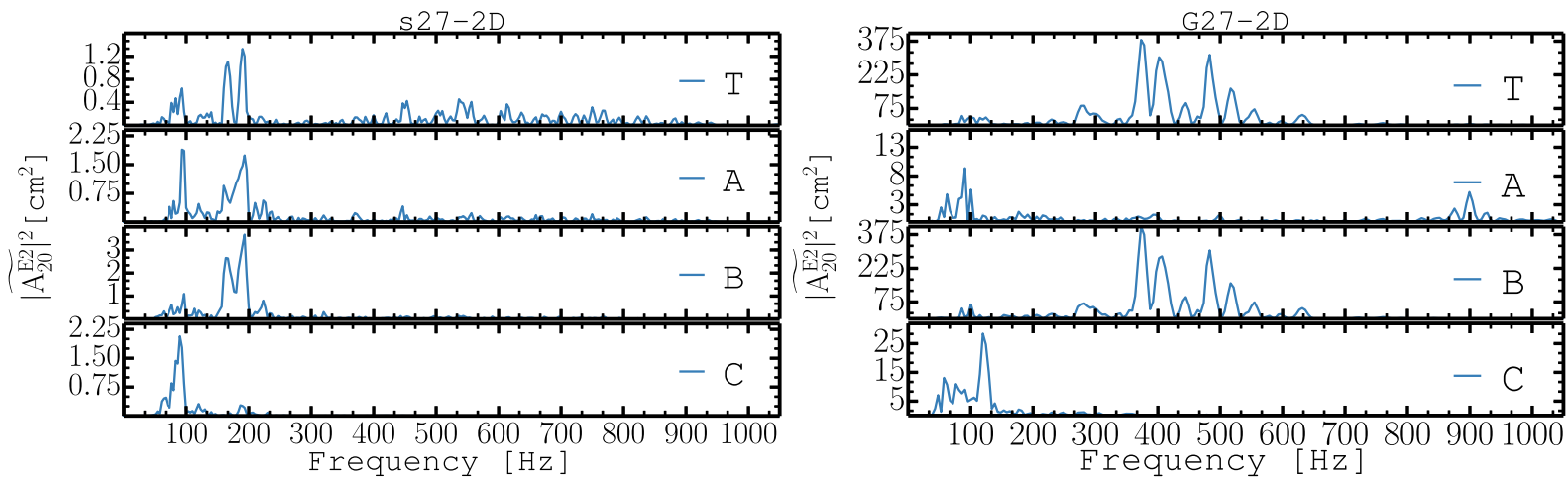

Figure 9. Squared Fourier amplitudes of the total volume-integrated GW signal and of the signal arising from the three different layers of the simulation volume, for the 2D model G27-2D of Müller et al. (2013) (right) and s27-2D (left). From the top: total signal (T), layer A, layer B, and layer C. The Fourier amplitudes, $\widetilde{\mathrm{A}_{E 2}^{20}}$, are calculated based on equations (14) and (11).

from model G27-2D of Müller et al. (2013) using the STFT and the decomposition of the computational volume into three different regions. G27-2D is a $2 \mathrm{D}$ model based on the same $27 \mathrm{M}_{\odot}$ progenitor used in our simulations, with the same EoS and the same neutrino treatment, the only major difference being the treatment of GR: in G27-2D, the equations of radiation hydrodynamics in the ray-by-ray-plus approximation are solved in their general relativistic formulation assuming a conformally flat metric, whereas the pseudo-Newtonian approach of Marek et al. (2006) was used for model s27 in 3D. The bottom row of Fig. 8 shows the GW signal and amplitude spectrogram for model G27-2D. Fourier amplitudes for the signal from the three regions are shown in right-hand panel of Fig. 9 for the period up to the onset of the explosion $210 \mathrm{~ms}$ after bounce. Comparing the spectrograms of model G27-2D with those of the 3D models (Figs 3 and 8), we find that the 'quiet zone' between the high-frequency and low-frequency components of the signal is not visible in model G27-2D, which is in agreement with the wavelet analysis of Müller et al. (2013) who also found a more broad-banded signal during phases of strong SASI and convection, with the Brunt-Väisälä frequency providing more of an upper limit rather than a sharply defined peak frequency during such phases. In model G27-2D, there is strong emission between 250 and $750 \mathrm{~Hz}$, and the relative contribution of the low-frequency signal below $250 \mathrm{~Hz}$ is smaller (which will be discussed in more detail in Section 3.6). The decomposition of the integration volume into three layers (left-hand panel of Fig. 9) reveals that the main contribution to the high-frequency signal stems from the PNS surface (layer B). In addition to the broad-band emission between 250 and $750 \mathrm{~Hz}$, 
there are also two narrow emission peaks centred around 800 and $900 \mathrm{~Hz}$. This emission is the result of oscillations deep in the PNS core that are excited by PNS convection.

Different from model G27-2D and other recent 2D models found in the literature (Marek et al. 2009; Murphy et al. 2009; Müller et al. 2013), a 2D version of model s27 (model s27-2D, simulated with PROMETHEUS-VERTEX) more closely resembles the 3D models presented in our study during the pre-explosion phase (i.e. up to $300 \mathrm{~ms}$ after bounce) in terms of its spectrogram (top right panel in Fig. 8) and time-integrated spectrum (left-hand panel of Fig. 9). The spectrogram of the model (top right panel of Fig. 8) shows the same two signal components that we found in our 3D models. There is a high-frequency and a low-frequency component and a frequency band separating the two components where the emission is much weaker (between 250 and $350 \mathrm{~Hz}$ ). The relative contributions to the total signal from layers A, B and C are roughly the same in models s27 and s27-2D (see left-hand panel of Fig. 9 and top left panel of Fig. 6). Judging from the time-integrated signals from the preexplosion phase, the only noteworthy difference between models s27 and s27-2D appears to consist in an overall reduction of the amplitudes by about a factor of 10 (or by a factor of 100 in squared amplitudes as shown in Figs 6 and 9) in 3D (s27) compared to s27-2D in all regions across the entire spectrum.

The fact that the signal from layer B is not very strong in s27-2D makes it difficult to determine the impact of 2D effects on mode excitations by motions in the gain region as opposed to motions in the PNS convection zone. The modes excited by motions in the gain region need to be very strong for the emission from layer B to clearly stick out in time-integrated spectra as in G27-2D. The spectrograms, however, show that the excitation of surface $\mathrm{g}$ modes by the SASI is more efficient in s27-2D than in the 3D model s27: in s27, significant emission in the high-frequency band is absent up to $\sim 210 \mathrm{~ms}$ after bounce despite strong SASI activity (which shows up in the lowfrequency band), whereas s27-2D shows noteworthy emission in the high-frequency band during this phase. In 3D, we thus find (i) a suppression of the signal originating from PNS convection (layer A) by a factor of $\sim 10$, and (ii) an even more efficient suppression of any high-frequency GW emission due to mode excitation by the SASI in the spectrograms.

There are presumably several reasons why the excitation of oscillations in the PNS surface layer is found to be more efficient in 2D than in 3D. First, the inverse turbulent cascade (Kraichnan 1967) and the suppression of the Kelvin-Helmholtz instability at the edge of supersonic downflows (see Müller 2015 and references therein) lead to an artificial accumulation of the turbulent energy on large scales in 2D supernova simulations (Hanke et al. 2012; Abdikamalov et al. 2015) and higher impact velocities of the downflows (Melson et al. 2015a; Müller 2015). Thus, both the amplitudes as well as the mode overlap of the forcing with the excited $l=2$ oscillation modes are higher in 2D. However, while the excitation of $g$ modes in the surface layer is strongly suppressed in 3D, there is still some residual g-mode activity (Melson et al. 2015a; Müller 2015). Furthermore, we must also consider the frequency structure of the forcing. Fig. 10 shows considerable high-frequency emission from layer $\mathrm{C}$ in $2 \mathrm{D}$, which is indicative of violent large-scale (i.e. with an $l=2$ component) mass motions on time-scales considerably shorter than the SASI period or the convective overturn time-scale. The lack of such high-frequency GW activity from layer $\mathrm{C}$ in 3D indicates that the downflows in $3 \mathrm{D}$ are not as strongly distorted by intermediate-scale eddies and that they vary less on short timescales. Fig. 4 of Melson et al. (2015a), which shows 2D and 3D simulations of a successfully exploding $9.6 \mathrm{M}_{\odot}$ model, further il-

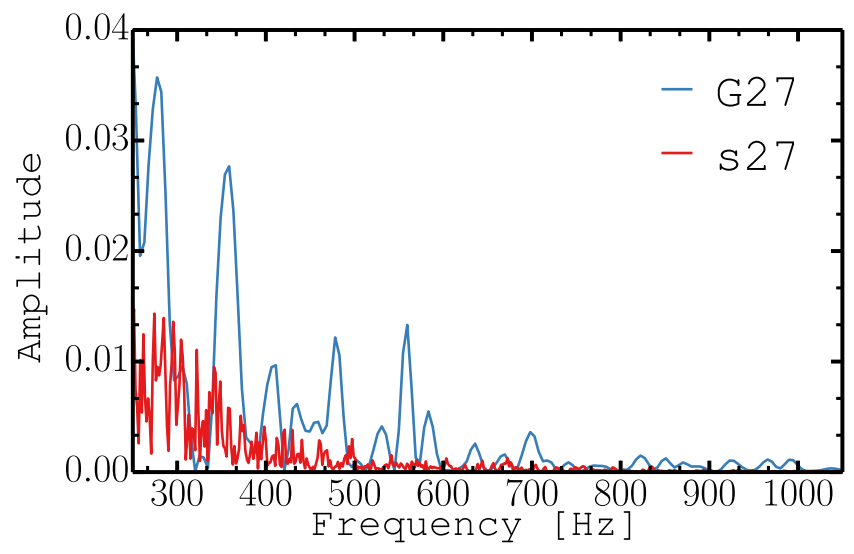

Figure 10. Normalized Fourier amplitudes of the GW signal from layer C for the 2D model G27-2D and the 3D model s27. Each curve is normalized by its respective maximum to account for the difference in magnitude between $2 \mathrm{D}$ and $3 \mathrm{D}$. Note that the maxima lie outside of the frequency domain shown in this figure.

lustrates this difference between the frequency structure of the postshock flow in 2D and 3D: in 3D the angle-averaged radial velocity profiles of the infalling material appear smooth. On the other hand, intermediate-scale eddies with fast time variations are clearly visible in 2D. With a typical time-scale of the order of $t \sim 1 \ldots 10 \mathrm{~ms}$, corresponding to a frequency range of $f \sim 100 \ldots 1000 \mathrm{~Hz}$, the eddies in $2 \mathrm{D}$ cause a more 'impulsive' forcing with a broad frequency spectrum. The different frequency structure of the forcing in 2D and $3 \mathrm{D}$ is then reflected in the excited PNS surface oscillations: in $2 \mathrm{D}$, where the frequency spectrum of the forcing overlaps with the natural g-mode frequency, we see resonant excitation of free (high-frequency) g-mode oscillations. This is not the case in $3 \mathrm{D}$, but we still see strong non-resonant excitation of forced g modes at low frequencies in the PNS surface (see Section 3.6).

It is noteworthy that some of these aforementioned effects (e.g. the different dynamics of supersonic downflows) depend on the Mach number of the flow and are therefore only relevant for the damping of the excitation of surface $\mathrm{g}$ modes by motions from the gain region. By contrast, Mach-number dependent effects like the inhibition of the Kelvin-Helmholtz instability in 2D will not affect the excitation of oscillation modes by PNS convection, which is strongly subsonic. This explains why 3D effects strongly quench g-mode excitation from SASI and hot-bubble convection, but lead to a more modest reduction of GW emission due to PNS convection, which therefore becomes the dominant source of high-frequency GW emission in our 3D models.

Without a 3D model corresponding to G27-2D, it is not yet possible to decide whether the suppression of surface g-mode excitation is generically strong enough to make it a subdominant source of GW emission. The critical feature in the post-shock flow that is responsible for strong GW emission from layer B in G27-2D is the development of very strong non-linear SASI activity with the presence of stable supersonic downflows, which reach Mach numbers of 1.5 already $110 \mathrm{~ms}$ after bounce. Since the coupling of perturbations in the gain region to the surface $g$ modes is more effective for high Mach number flow (cp. Goldreich \& Kumar 1990; Lecoanet \& Quataert 2013), and since the kinetic energy in non-radial motions reaches large values of up to $6 \times 10^{49} \mathrm{erg}$ (compared to preexplosion values of $\lesssim 1.5 \times 10^{49}$ erg in s27 and s27-2D, cp. Hanke et al. 2013), the resulting GW amplitudes from surface $g$ modes in G27-2D are one order of magnitude larger than in s27-2D and two 


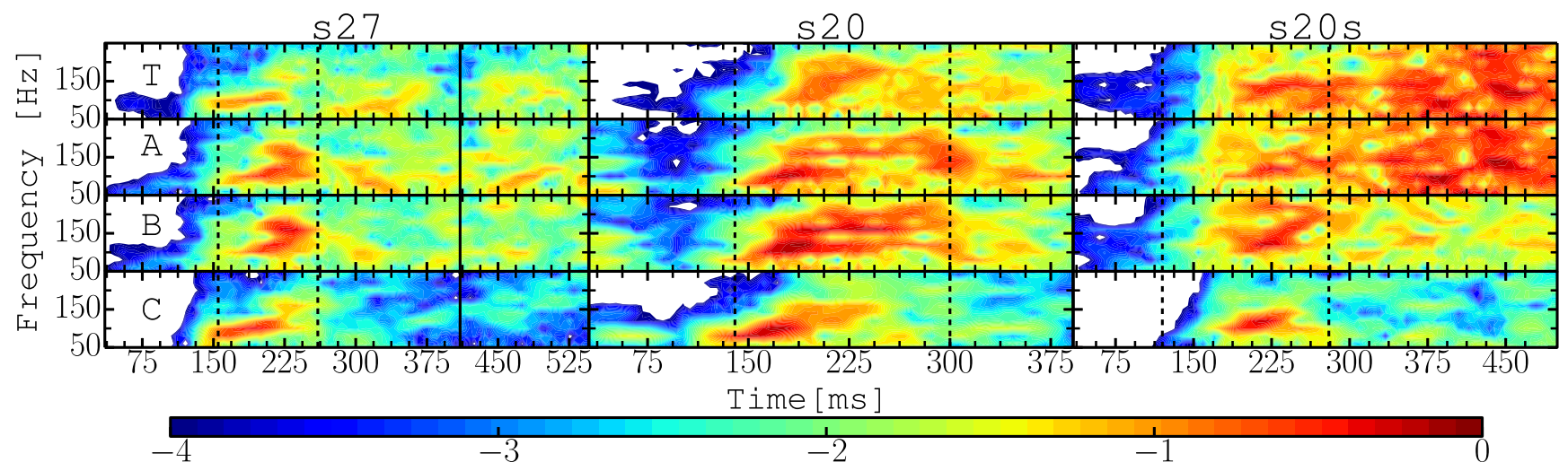

Figure 11. Amplitude spectrograms, for a sliding window of $50 \mathrm{~ms}$, of the low-frequency GW signal arising from the three different layers, summed over the two polarization modes $\left(\left|\widetilde{A}_{+}\right|^{2}+\left|\widetilde{A}_{\times}\right|^{2}\right)$. From the top: total signal (T), layers A, B and C. Columns are ordered by progenitor (left: s27, middle: s20, right: s20s). See Fig. 4 for a sketch of the three regions. The observer is chosen to be the north pole in the computational grid. As in Figs 1 and 3 , strong SASI episodes are bracketed by vertical lines. The colour scale is logarithmic and all panels have been normalized by the same global factor that has also been used for Fig. 3 .

orders of magnitude larger than in s27, which only develops mildly non-linear SASI activity during the pre-explosion phase. The GW emission from layer B thus clearly dominates the time-integrated spectrum of G27-2D.

Under the conditions that obtain in model G27-2D (fully developed non-linear SASI/convection with high Mach numbers), 2D/3D differences in the behaviour of the downflows due to the inhibition of the Kelvin-Helmholtz instability in 2D tend to become more pronounced, which suggests that very little excitation of surface $g$ mode by the SASI may survive in 3D even for a model with similar dynamics to G27-2D. This will need to be confirmed by future 3D simulations, however.

\subsection{Origin of the low-frequency signal}

The strong low-frequency signal seen in the more massive models (s20, s20s and s27) is apparently closely connected to SASI activity. Note that the convection-dominated s11.2 model shows some stochastic low-amplitude GW emission at low frequencies (see Fig. 3), which is, however, much less pronounced compared to the high-frequency component. To address the origin of the lowfrequency component, we show spectrograms of the GW signal below $250 \mathrm{~Hz}$ from each of the three analysis regions for models s20, s20s and s27 in Fig. 11.

The apparent temporal correlation of the low-frequency emission with the SASI suggests the following plausible mechanism responsible for this component: violent SASI involves the development of large-scale, large-amplitude density perturbations with the same temporal dependence as the shock oscillations. Such density perturbations $\delta \rho$ will directly contribute to the signal through the term $\rho x_{i} \partial_{j} \Phi$ in the integrand of the quadrupole formula (6) if the density perturbations $\delta \rho$ have an $l=2$ (quadrupole) component $\delta \rho_{2} .{ }^{4}$ Even $l=1$ sloshing and spiral motions will develop a sizeable quadrupole component $\delta \rho_{2}$ in the non-linear phase. The frequency of the emitted signal will trace the frequency of the underlying SASI mode, but with frequency doubling for the $l=1$ mode since the SASI-induced perturbation of the quadrupole moment will repeat itself after half

\footnotetext{
${ }^{4}$ Velocity perturbations will, in principle, also contribute in equation (6) Empirically, we find that their contribution to the GW amplitude is minimal, however.
}

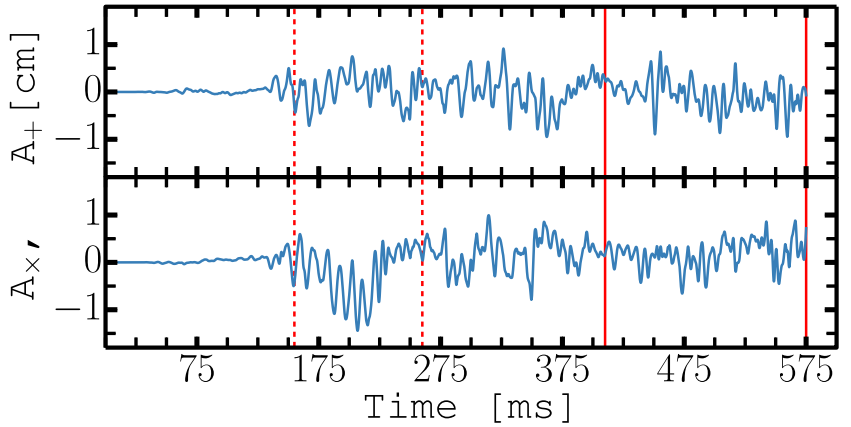

Figure 12. The GW amplitudes $A_{+}$and $A_{\times}$of model s 27 , for an observer situated along the $z$-axis of the computational grid, after a low-pass filter has been applied. The time axis indicates time since core bounce.

a period as the integrand in equation (6) is invariant to a rotation by $\pi$ in any direction. The contribution of the $l=1$ and $l=2$ modes (and possibly their overtones) explains the double-peak structure of the low-frequency signal in Figs 3, 6 and 11. The amplitude arising from the term $\rho x_{i} \partial_{j} \Phi$ will be of the order of

$A \sim \frac{G}{c^{4}} \int r \delta \rho_{2} \frac{\partial \Phi}{\partial r} \mathrm{~d} V \sim \frac{G}{c^{4}} \int \delta \rho_{2} \frac{G M}{r} \mathrm{~d} V$.

The integral is essentially the potential energy stored in $l=2$ density perturbations during SASI oscillations. Equating the potential energy with the kinetic energy in SASI motions and taking into account that there is only a finite overlap with $l=2$, we find

$A \lesssim \frac{G}{c^{4}} E_{\text {kin,SASI }}$

With $E_{\text {kin,SASI }} \sim 10^{49} \mathrm{erg}$, we obtain $A \lesssim 0.8 \mathrm{~cm}$, which is roughly compatible with the amplitudes (see Fig. 12).

The anisotropic modulation of the accretion by the SASI is further communicated to the PNS as material is advected downwards and settles on to the PNS surface (something which may also be viewed as non-resonant excitation of $\mathrm{g}$ modes far below their eigenfrequency). As matter seeps deeper into the outer layer of the PNS (layer B) and then even further down into the interior of the PNS (layer A), it will still emit GWs if the density and entropy perturbations are not washed out completely by neutrino cooling. We have verified that relatively large density fluctuations on the percent level are maintained even in the cooling region. Since these density 
fluctuations still retain a temporal modulation set by the SASI, they emit GWs in a similar, albeit somewhat broader frequency range. For the same reasons as detailed above, the GWs amplitudes produced by such a non-resonant excitation of $\mathrm{g}$ modes will be related to the kinetic energy stored in the mode and even a small kinetic energy $\gtrsim 10^{48} \mathrm{erg}$ in aspherical mass motions below the gain region is sufficient to account for the amplitudes.

The fact that the low-frequency signal from layer $\mathrm{C}$ is weaker than that from both layer A and layer B is not in conflict with this explanation because of cancellations in the integral of $\rho\left(v_{i} v_{j}-x_{i} \partial_{j} \Phi\right)$ over the region outside the PNS, e.g. the overdensities in the downflows can be compensated by the smaller shock radius above them. ${ }^{5}$ Furthermore, we surmise that density perturbations from the $l=1$ contribute more strongly to the GW signal as they settle deeper into the PNS, because the pure $l=1$ angular dependence of the perturbations in the post-shock region develops a larger $l=2$ component during the process of settling.

The crucial role of the SASI in providing a slow, non-resonant forcing of the outer regions of the PNS is also reflected in the frequency structure of the signal. In Fig. 13, we plot the Fourier amplitudes of the $l=1$ and $l=2$ components of the spherical harmonics decomposition of the shock position for the period between 100 and $350 \mathrm{~ms}$ after bounce. More precisely Fig. 13 shows

$\sum_{m=-l, l}\left|\widetilde{a}_{l}^{m}(t)\right|^{2}(l=1,2)$,

where $\widetilde{a}_{l}^{m}(t)$ is the Fourier transform of

$a_{l}^{m}\left(t_{n}\right)=\frac{(-1)^{|m|}}{\sqrt{4 \pi(2 l+1)}} \int r_{\mathrm{sh}}(\theta, \phi, t) Y_{l}^{m} \mathrm{~d} \Omega$.

Here, $r_{\mathrm{sh}}$ is the shock position (given by the Riemann-solver in our code) and $Y_{l}^{m}$ is the spherical harmonic of degree $l$ and order $m$. Details about the shock can be found in Hanke et al. (2013) for model s27, in Hanke (2014) for models s11.1 and s20 and in Melson et al. (2015b) for model s20s. The typical frequency for the $l=1$ mode $(50 \ldots 100 \mathrm{~Hz})$ and the $l=2$ mode $(100 \ldots 160 \mathrm{~Hz})$ of the shock is compatible with the range of low-frequency emission seen in the GW spectrograms, especially if we account for the fact that the GW signal from forced $l=1$ motion will exhibit frequency doubling.

Since the Fourier spectra of the $l=1$ and $l=2$ modes as well as the GW spectrogram point towards a complicated frequency structure with peak frequencies shifting in time (due to the variation of the shock radius which sets the SASI frequencies) and contributions from different phases interfering with each other in the time-integrated spectrum, we refrain from a precise one-to-one identification of the underlying modes.

It is noteworthy that the effect of anisotropic accretion manifests itself even down to the PNS convective layer. Apparently, the eigenfunctions of the excited modes reach down quite deep through the entire surface of the PNS (layer B). However, the fact that even the deeper region of layer A (below the overshooting region) contributes to low-frequency GW emission suggests that the $l=1$ and $l=2$ surface motions can trigger convective motions (e.g. by providing density perturbations that are then quickly amplified once they are advected into the convectively unstable region). Contrary

\footnotetext{
${ }^{5}$ Immediately outside the minimum shock radius, the densities of unshocked material above the downflows are lower than in the shocked material inside the high-entropy bubbles at a given radius, i.e. overdensities behind the shock correspond to underdensities at larger radii.
}
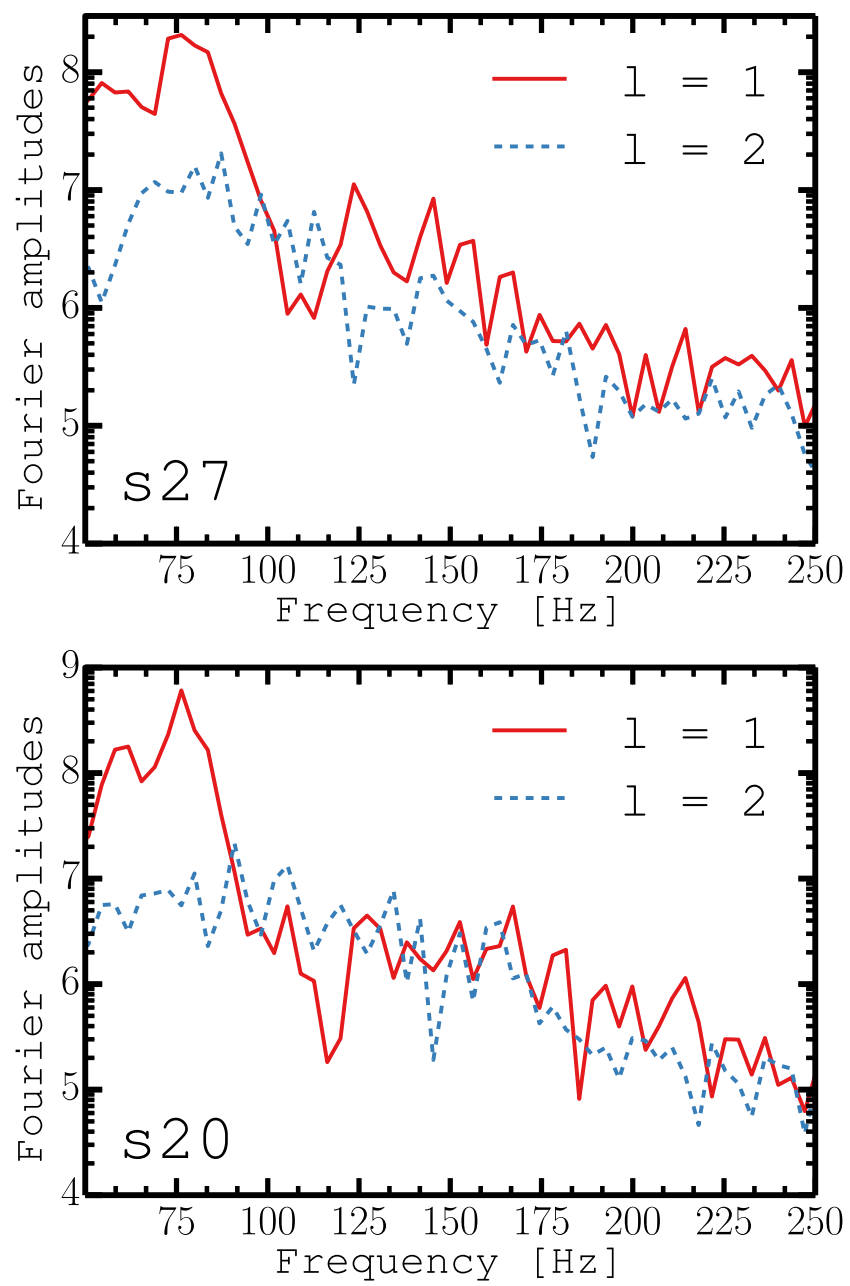

Figure 13. Squared Fourier amplitudes, in logarithmic scale, for the $l=1$ and $l=2$ components of the expansion of the shock position into spherical harmonics. The Fourier amplitudes have been calculated for the time window between 100 and $350 \mathrm{~ms}$ after core bounce. The upper panel shows model s27 and the bottom panel shows the results for model s20. The curves in both panels have been normalized by the same factor.

to the mirror problem of wave excitation at convective boundaries (Goldreich \& Kumar 1990; Lecoanet \& Quataert 2013), such a coupling between the accretion flow, the surface layer and the PNS convection has as yet been poorly explored.

While the SASI is particularly effective at generating a modulation of the accretion flow with a sizeable $l=2$ component, largescale convective motions in the hot-bubble region can also act as a substitute for the SASI during periods of transient shock expansion (because the typical scale of convective eddies is set by the width of the unstable region, cp. Chandrasekhar 1961; Foglizzo, Scheck $\&$ Janka 2006). The result is a somewhat weaker and less sharply defined low-frequency signal, which is what we observe during the SASI-quiet periods in models s20s, s20 and s27 and also in model s11.2 (cf. Fig. 3).

With large-scale fluid motions in the gain region as the ultimate agent responsible for low-frequency GW emission (through forced PNS oscillations), the temporal structure of this signal component finds a natural explanation. Generally, episodes of strong SASI activity correlate with strong low-frequency GW activity. Large amplitudes of the shock oscillations are not sufficient; however, 


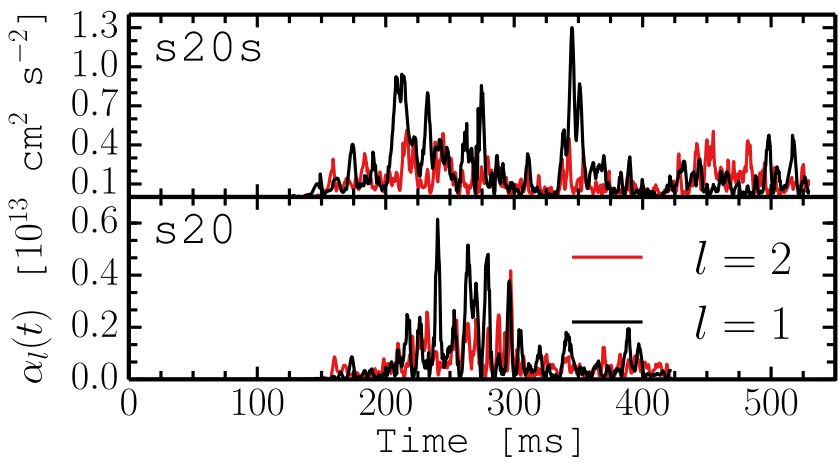

Figure 14. The $l=2$ and $l=1$ components of the radial velocity sampled in the unstable PNS layer for the $20 \mathrm{M}_{\odot}$ models. The radial velocity has been sampled at a radius $R$ given by $\rho(R)=9.5 \times 10^{13} \mathrm{~g} \mathrm{~cm}^{-3}$. The top panel shows the exploding model s20s, and the bottom panel shows the non-exploding model s20, which was only calculated to $421 \mathrm{~ms}$ after core bounce.

the determining factor is the kinetic energy contained in large-scale motions. For that reason, there is hardly any low-frequency emission component during the second SASI episode in model s27. During this phase, less mass is involved in SASI motions and the SASI amplitude is significantly smaller. The lack of large-scale motions with a significant $l=2$ component also explains the weak lowfrequency GW activity in model s11.2, where the post-shock flow is dominated by smaller convective bubbles and the kinetic energy in non-radial fluid motions is typically smaller than for the more massive progenitors.

\subsection{Comparison of the exploding and non-exploding 20 solar mass models}

The exploding model s20s differs only in details from its nonexploding counterpart during the accretion phase. After the onset of shock expansion, strong low-frequency emission is sustained until the end of the simulation (see Fig. 11). This emission is connected to mass motions with a strong $l=2$ component in layer A. In Fig. 14, we plot for models s20 and s20s,

$\alpha_{l}=\sum_{m=-l}^{l}\left|\alpha_{l}^{m}(t)\right|^{2},(l=1,2)$,

with

$\alpha_{l}^{m}(t)=\frac{(-1)^{|m|}}{\sqrt{4 \pi(2 l+1)}} \int v_{\mathrm{r}}(\theta, \phi, t) Y_{l}^{m} \mathrm{~d} \Omega$,

where $v_{\mathrm{r}}$ is the radial velocity at a radius $R$ corresponding to a spherically averaged density of $\rho(R)=9.5 \times 10^{13} \mathrm{~g} \mathrm{~cm}^{-3}$.

In the exploding model, the $l=2$ mode is generally stronger than in the non-exploding model and it remains strong throughout the simulation in contrast to the non-exploding model, where the $l=2$ mode decreases in strength after the SASI-dominated phase ends. After a period of decreasing strength around $400 \mathrm{~ms}$, the quadrupole mode in model s20s increases in strength and reaches amplitudes similar to those seen during the pre-explosion phase. At the same time, there is a shift in the relative strength of the $l=1$ and $l=2$ mode after the onset of shock expansion, while the quadrupole mode increases in strength, the dipole is relatively weak at late times. This transition into a flow pattern that is dominated by an $l=2$ mode resonates better with the quadrupole nature of $\mathrm{GW}$ emission. We therefore see an increase in low-frequency emission from the unstable layer within the PNS. Such a change in the spectrum of eddy scales after shock revival could result from changes in the asymmetric accretion flow on to the PNS, or from changes in the stratification of entropy and electron fraction, but for the purpose of interpreting the GW emission, the ultimate reason is immaterial and left to more detailed future studies of the hydrodynamics of PNS convection. Aliasing of high-frequency emission may also be partly responsible for the enhanced low-frequency emission after shock revival. However, since the spectrograms in Fig. 3 show broad-band emission, it is unlikely that the low-frequency emission we see after shock revival is caused by aliasing effects alone.

\section{DETECTION PROSPECTS}

With the prominent high-frequency component of the signal in 2D largely muted in $3 \mathrm{D}$, it is evidently necessary to reconsider the prospects of detecting a Galactic supernova. Detailed detectability studies based on 2D waveforms (Logue et al. 2012) may now well be too optimistic after the update of the waveform predictions. While an elaborate statistical machinery is required to reliably determine signal detectability and possible inferences about core-collapse supernova physics (Logue et al. 2012; Hayama et al. 2015), we can already draw some conclusions for the waveforms presented in this paper.

\subsection{General considerations}

The detectability of the GW signal from a core-collapse supernova has often been assessed using the signal-to-noise ratio (SNR) for matched filtering. Assuming an optimally orientated detector and a roughly isotropic frequency spectrum for different observer directions, the SNR for matched filtering is formally given by (Flanagan \& Hughes 1998, cp. their equation 5.2 for the second form),

$(\mathrm{SNR})^{2}=4 \int_{0}^{\infty} \mathrm{d} f \frac{|\tilde{h}(f)|^{2}}{S(f)}=\int_{0}^{\infty} \mathrm{d} f \frac{h_{c}^{2}}{f^{2} S(f)}$,

where

$h_{c}=\sqrt{\frac{2 G}{\pi^{2} c^{3} D^{2}} \frac{\mathrm{d} E_{\mathrm{GW}}}{\mathrm{d} f}}$

is the characteristic strain, $S(f)$ is the power-spectral density of the detector noise as a function of frequency $f$ and $\mathrm{d} E_{\mathrm{GW}} / \mathrm{d} f$ is the spectral energy density of the GWs. Note that the second expression for the SNR in equation (27) has been obtained under the assumption of isotropic GW emission so that one can express the (formally) direction-dependent squared amplitudes in terms of the GW energy spectrum $\mathrm{d} E_{\mathrm{GW}} / \mathrm{d} f$.

Since the GW signal of a core-collapse supernova is, however, not amenable to matched filtering because of its stochastic character, the SNR formally defined by equation (27) must be interpreted with care.

The SNR still remains a useful quantity as it measures the excess power during the time of integration, as can be seen by re-expressing equation (27) in terms of the expectation value for the Fourier coefficients $\tilde{n}(f)$ of the noise over a finite time-interval $\Delta t$ (the integration time for the signal), which obey (cp. Logue et al. 2012)

$\left\langle\tilde{n}(f) \tilde{n}^{*}(f) \Delta f\right\rangle=S(f) / 2$,

where the factor $1 / 2$ appears because $S(f)$ is defined as the one-sided power spectral density of the time-dependent strain noise $n(t)$. Note that the frequency spacing $\Delta f$ is given by $\Delta f=1 / \Delta t$ and that $\Delta t$ 
Table 1. SNR for all four models. Values are given for two different frequency domains, $20 \ldots 250 \mathrm{~Hz}$ (low frequency) and $250 \ldots 1200 \mathrm{~Hz}$ (high frequency). The table shows values for two different detectors, AdvLIGO and the Einstein Telescope. For the latter, we calculate the SNR for two different modes of operation (ET-B and ET-C). SNRs have been computed for a source at a distance of $10 \mathrm{kpc}$. For model s20s, we only show the SNR for the low-frequency band since the high-frequency band is somewhat contaminated by aliasing effects. For s27, s20 and s11.2 we also give the ratio of the band-limited SNRs in the low- and high-frequency bands to quantify the 'colour' of the GW signal.

\begin{tabular}{|c|c|c|c|c|c|c|c|c|c|c|c|c|c|c|c|}
\hline & \multicolumn{4}{|c|}{ s27 } & \multicolumn{4}{|c|}{ s20 } & \multicolumn{3}{|c|}{$\mathrm{s} 20 \mathrm{~s}$} & \multicolumn{4}{|c|}{$\mathrm{s} 11.2$} \\
\hline & Low & High & Total & Low/High & Low & High & Total & Low/High & Low & High & Total & Low & High & Total & Low/High \\
\hline AdvLIGO & 3.7 & 4.5 & 8.8 & 0.82 & 5.3 & 7.7 & 9.4 & 0.82 & 10.2 & - & - & 1.3 & 4.1 & 4.3 & 0.32 \\
\hline ET-B & 78.5 & 73.7 & 107.7 & 1.07 & 113.9 & 127.0 & 170.6 & 0.74 & 217.3 & - & - & 28.0 & 67.3 & 72.8 & 0.42 \\
\hline
\end{tabular}

can be set to the length of the signal in consideration in our case. For a finite time series, where the integral in equation (27) can be replaced with a sum over the Fourier modes at discrete frequencies $f_{k}=k / \Delta t$ (with integer $k$ ), we then obtain,

$(\mathrm{SNR})^{2}=8 \sum_{k} \frac{\left|\tilde{h}\left(f_{k}\right)\right|^{2} \Delta f}{\left\langle\left|\tilde{n}\left(f_{k}\right)\right|^{2}\right\rangle \Delta f}=8 \sum_{k} \frac{\left|\tilde{h}\left(f_{k}\right)\right|^{2}}{\left\langle\left|\tilde{n}\left(f_{k}\right)\right|^{2}\right\rangle}$.

For uncorrelated Gaussian noise in each frequency bin, the SNR of a prospective signal obtained from the summation over $N_{\text {bins }}$ frequency bins is thus related to the $\chi^{2}$-value for this signal as

$\chi^{2} \sim N_{\text {bins }}+\mathrm{SNR}^{2} / 2$,

where the additional term $N_{\text {bins }}$ comes from the contribution of the noise in each bin. Sufficiently high values of $\chi$ during a prospective supernova event (with an integration interval $\Delta t$ defined by a coincident neutrino signal) ${ }^{6}$ can be attributed to a physical signal; e.g. to exclude stochastic fluctuations as a source of the excess power at a confidence level of 95 per cent, one needs

$\mathrm{SNR}^{2} / 8=\chi^{2}-N_{\text {bins }} \gtrsim 2.3 \sqrt{N_{\text {bins }}}$

for large $N_{\text {bins }}$. For a signal with power excess in a frequency band with bandwidth $\delta f$ and $N_{\text {bins }}=\delta f / \Delta f=\delta f \Delta t$, this implies the requirement

$\mathrm{SNR} \gtrsim 4.3(\delta f \Delta t)^{1 / 4}$,

for a detection of a signal in this band. This roughly corresponds to the results obtained by Flanagan \& Hughes (1998) for noise monitoring in section IIB of their paper.

Prior knowledge of the signal structure can help to identify signals with even lower SNR; Logue et al. (2012), for example, showed that a detection and identification can be possible already for SNR $\sim 10$ with the help of a principal component analysis of template waveforms provided that the signal structure is not too dissimilar from the template. This is in line with the weak dependence of equation (33) on the bandwidth $\delta f$.

If properly interpreted, the SNR thus remains a useful measure for the detectability of our predicted signals within the scope of this paper. Its inherent limitations provide justification for neglecting the effect of the detector orientation and the precise directional dependence of the signal by computing the SNR from the energy spectrum $\mathrm{d} E / \mathrm{d} f$ instead of a direction-dependent Fourier spectra of the strain. We have verified that the SNR for the low-frequency band does not vary by more than $\sim 20$ per cent, and the SNR

\footnotetext{
${ }^{6}$ This is crucial because it is always possible to find short intervals with power excess comparable to a physical signal if the integration time is sufficiently long.
}

for the high-frequency signal depends even less on the observer direction.

\subsection{Detection prospects for simulated models}

We calculate the SNR from equation (27) for the zero-detuning high power configuration of Advanced LIGO (LIGO Laboratory \& Shoemaker 2010) and the B (Hild, Chelkowski \& Freise 2008) and C (Hild et al. 2010) configurations for the Einstein Telescope. We refer to these configurations as AdvLIGO, ET-B and ET-C. In order to better assess the detectability and possible inferences from the signal structure, we compute SNRs quantifying the excess power in a low-frequency band $\left(\mathrm{SNR}_{\text {low }}\right.$ for $20 \mathrm{~Hz} \leq f<250 \mathrm{~Hz}$, i.e. $\delta f=230 \mathrm{~Hz})$ and a high-frequency band $\left(\mathrm{SNR}_{\text {high }}\right.$ for $250 \mathrm{~Hz} \leq$ $f<1200 \mathrm{~Hz}$, i.e. $\delta f=950 \mathrm{~Hz}$ ). SNRs for all models in those two bands for events at a distance of $10 \mathrm{kpc}$ are presented in Table 1. Using equation (33), we obtain a detection threshold of $\mathrm{SNR}_{\text {low }} \gtrsim 11$ for the low-frequency band and $\mathrm{SNR}_{\text {high }} \gtrsim 15$ for the high-frequency band assuming $\Delta t=0.5 \mathrm{~s}$. Since the critical SNR depends weakly on $\Delta t$, these fiducial values can be used for all models. SNRs for arbitrary distances can easily be obtained since the SNR is inversely proportional to the distance.

Regardless of the precise detector configuration, the SASIdominated models s20 and s27 are clearly distinguished from the convective model s11.2 through a higher ratio $\mathrm{SNR}_{\text {low }} / \mathrm{SNR}_{\text {high }}>0.65$ compared to $\mathrm{SNR}_{\text {low }} / \mathrm{SNR}_{\text {high }}<0.42$. SASI-dominated models thus appear 'redder' in GWs before the onset of the explosion. Based on our small sample, they also appear to be characterized by a higher SNR, but this might be incidental. More massive progenitors with stronger neutrino heating in the gain region, stronger cooling above the PNS convection, and a larger mass in the gain region could produce a stronger GW signal, even in the absence of strong SASI activity. The ratio $\mathrm{SNR}_{\text {low }} / \mathrm{SNR}_{\text {high }}$, on the other hand, should be a robust indicator for the presence or absence of large-scale SASI motions.

Note that since model s20s suffers most severely from aliasing effects, the SNR in the high-frequency domain might be inaccurate. We therefore refrain from giving values for $\mathrm{SNR}_{\text {high }}$ and the total SNR. The low-frequency band, on the other hand, should be unaffected by aliasing artefacts and $\mathrm{SNR}_{\text {low }}$ is significantly higher than in the non-exploding models. It is possible that the enhanced low-frequency emission from the convectively unstable region of the PNS is a general feature in exploding models and we hypothesize that shock revival will be followed by GW emission with excess power in the low-frequency band. This is in contrast to previous studies in 2D (Murphy et al. 2009; Müller et al. 2013) where shock expansion is typically followed by an increase in the high-frequency emission band. If shock revival generally leads to enhanced low-frequency emission, this would obviously complicate 
the interpretation of a high value of $\mathrm{SNR}_{\text {high }} / \mathrm{SNR}_{\text {low }}$, which could either indicate SASI activity or the transition to an explosion.

\subsection{Detection prospects with AdvLIGO}

For a supernova at a distance of $10 \mathrm{kpc}$, it is evident that none of the four models could be detected by AdvLIGO based on excess signal power. Given the reduction of the typical amplitudes by a factor of $\sim 10$ in $3 \mathrm{D}$ compared to $2 \mathrm{D}$, this is not surprising. Using an approach based on simulated noise and a principal component analysis of the signal, Logue et al. (2012) and Gossan et al. (2016) already found that AdvLIGO is only marginally able to identify waveforms from 2D supernova simulations for events at distances of a few kpc.

For the SASI-dominated models (s20, s27, s20s), the excess power in the low-frequency band would become detectable at 95 per cent confidence level at the distance of the Crab supernova ( $\sim 2 \mathrm{kpc}$ ), as would the high-frequency component of model s20. Model s11.2, on the other hand, would not show a statistically significant power excess.

\subsection{Detection prospects with the Einstein Telescope}

The situation will change drastically with the Einstein Telescope. For either configuration considered here, the excess power in both bands ought to be detectable for an event at a distance of $10 \mathrm{kpc}$, although the low-frequency component of model s11.2 would barely make it above the detection threshold for ET-C. The high SNR in both bands would permit a measurement of $\mathrm{SNR}_{\text {low }} / \mathrm{SNR}_{\text {high }}$ as an indicator for the GW 'colour' with some confidence. Even at a distance of $20 \mathrm{kpc}$, the excess power in both the high- and lowfrequency bands would still remain detectable and quantifiable in the SASI-dominated models. For the more modest goal of a mere detection, the SNR for model s20s would be high enough to observe events throughout the entire Milky Way and even out to the Large Magellanic Cloud ( $~ 50 \mathrm{kpc})$.

\subsection{Interpretation of a prospective detection}

Without a more sophisticated analysis of the time-frequency structure of a prospective detection event, only limited conclusions about the supernova core could be drawn from excess power measured by GW detectors during specific time windows. None the less, a GW detection with the Einstein Telescope would be valuable for corroborating our understanding of hydrodynamic instabilities in the core in conjunction with the observed neutrino signal.

A high value of $\mathrm{SNR}_{\text {low }} / \mathrm{SNR}_{\text {high }}$ concurrent with a periodic modulation of the neutrino signal (Marek et al. 2009; Lund et al. 2010; Brandt et al. 2011; Tamborra et al. 2013, 2014a; Müller \& Janka 2014) would furnish solid evidence for SASI activity, and strong low-frequency emission concurrent with modulations of the neutrino signal below $\sim 50 \mathrm{~Hz}$ would strongly indicate that shock revival is already underway during the time window in question. While these conclusions could likely be drawn on the basis of the neutrino signal alone for nearby supernovae with a suitable orientation of the SASI spiral plane or sloshing mode, the detection of modulations in the neutrino signal for non-optimal orientations becomes difficult at distances $\gtrsim 10 \mathrm{kpc}$ (Müller \& Janka 2014). In such cases, combining the $\mathrm{GW}$ and neutrino signal would likely allow stronger conclusions.

When SASI-induced modulations of the neutrino signal are not detectable due to distance, orientation or unfavourable neutrino flavour oscillations, a detection of strong GW power in the lowfrequency band would still provide evidence for either SASI activity (since this signal component is more robust against orientation effects than modulations of the neutrino signal) or the onset of strongly asymmetric accretion after shock revival. If the SNR is sufficiently high to localize the GW power excess in time relative to the onset of the neutrino signal (which roughly marks the time of bounce), it may be possible to decide between those two alternatives.

Late GW power excess after $\gtrsim 0.5 \mathrm{~s}$ will likely indicate the onset of the explosion without prior SASI activity, since the SASI typically reaches non-linear saturation well before this point, and since the decreasing mass in the gain region does not allow for strong late-time GW emission due to the SASI (as shown by models s20 and s27).

\section{CONCLUSIONS}

We have studied the GW signal from the accretion phase and the early explosion phase of core-collapse supernovae based on four recent 3D multigroup neutrino hydrodynamics simulations. We considered four models based on three progenitors with ZAMS masses of 11.2, 20 and $27 \mathrm{M}_{\odot}$. The three non-exploding models enabled us to study the phase between bounce and shock revival. We covered both the SASI-dominated regime (model s20, Tamborra et al. 2014a; model s27, Hanke et al. 2013), as well as the convection-dominated regime (model s11.2, Tamborra et al. 2014b). Additionally, the exploding $20 \mathrm{M}_{\odot}$ model s20s (Melson et al. 2015b, with a modified axial-vector coupling constant for neutral current scattering) illustrates changes in the GW signal in exploding models. Since our treatment of the microphysics and the neutrino transport is on par with previous works on the GW signal from 2D simulations (Marek et al. 2009; Yakunin et al. 2010; Müller et al. 2013; Yakunin et al. 2015), we were in the position to conduct a meaningful comparison of GW emission in 2D and 3D during the accretion and explosion phase for the first time. To this end, we included the $27 \mathrm{M}_{\odot}$ 2D models of Müller et al. (2012b) and Hanke et al. (2013) in our study.

Our analysis showed differences between the GW emission in $2 \mathrm{D}$ and $3 \mathrm{D}$. The prominent, relatively narrow-banded emission at high frequencies that is characteristic of $2 \mathrm{D}$ models is significantly reduced. With the reduction of the high-frequency emission, distinctive broad-band low-frequency emission in the range between 100 and $200 \mathrm{~Hz}$ emerges as a characteristic feature during episodes of SASI activity and during the explosion phase of model s20s. The low-frequency emission does also exist in the $2 \mathrm{D}$ models, but it is completely overwhelmed by the highfrequency emission. This conclusion is somewhat model dependent, because in one of our 2D models, s27-2D, high-frequency GW emission is low and the low-frequency component becomes very prominent.

We discussed these differences extensively from two vantage points: on the one hand, we investigated the underlying hydrodynamic processes responsible for GW emission and showed how the changes in the GW signal in 3D are related to critical differences in flow dynamics in 3D compared to $2 \mathrm{D}$. On the other hand, we outlined the repercussions of these changes for future $\mathrm{GW}$ observations and sketched possible inferences that could be drawn from the detection of a Galactic event by third-generation instruments.

With regard to the hydrodynamic processes responsible for GW emission, our findings can be summarized as follows: 
(i) There is a high-frequency signal component that closely traces the buoyancy frequency in the PNS surface region in 2D and 3D, i.e. the roughly isothermal atmosphere layer between the PNS convection zone and the gain region acts as a frequency stabilizer for forced oscillatory motions in both cases. However, the highfrequency component mostly stems from aspherical mass motions in and close to the overshooting region of PNS convection in 3D, whereas it stems from mass motion close to the gain radius in 2D. This indicates that quasi-oscillatory mass motions at high frequencies are instigated only by PNS convection in $3 D$ even during the pre-explosion phase, whereas forcing by the SASI and convection in the gain region is dominant in 2D. The resulting amplitudes of the high-frequency component are considerably lower in 3D than in $2 \mathrm{D}$.

(ii) We ascribe the strong excitation of high-frequency surface g-mode oscillations in 2D to several causes: the inverse turbulent cascade in 2D leads to larger impact velocities of the downflows and creates large flow structures that can effectively excite $l=2$ oscillations that give rise to $\mathrm{GW}$ emission. Braking of downflows by the forward turbulent cascade and fragmentation into smaller eddies strongly suppress surface g-mode excitation in 3D. Moreover, the spectrum of turbulent motions does not extend to high frequencies in 3D both in SASI-dominated and convection-dominated models so that the resonant excitation of the $l=2$ surface $g$ mode at its eigenfrequency becomes ineffective.

(iii) In 3D, low-frequency $\mathrm{GW}$ emission in the pre-explosion phase ultimately stems from the global modulation of the accretion flow by the SASI. Because of frequency doubling and/or the contribution from the $l=2$ mode, the typical frequencies of this component are of the order of $100 \ldots 200 \mathrm{~Hz}$, i.e. somewhat higher than the typical frequency of the $l=1$ modes of the SASI. Mass motions in the post-shock region, the PNS surface region and the PNS convection zone all contribute to this low-frequency component, which indicates that the modulation of the accretion flow is still felt deep below the gain radius as the accreted matter settles down on to the PNS. Moreover, our analysis of the detection prospects shows that the low-frequency component of the signal at $\gtrsim 100 \mathrm{~Hz}$ becomes a primary target in terms of detectability in contrast to previous $2 \mathrm{D}$ results.

(iv) By contrast, convective models characterized by mass motions of intermediate- and small-scale-like s11.2 show very little GW emission at low frequencies. The high-frequency emission, on the other hand, is excited primarily by PNS convection and is therefore less sensitive to the dominant instability (convection or SASI) in the post-shock region. Thus, the ratio of high-frequency to low-frequency GW power can potentially be used to distinguish SASI- and convection-dominated models in the pre-explosion phase.

(v) However, strongly enhanced low-frequency emission can also occur due to a change of the preferred scale of the convective eddies in the PNS convection zone as exemplified by model s20s, where the dominant mode shifts from $l=1$ to $l=2$ late in the simulation. Since this does not occur in the corresponding non-exploding model s20, one can speculate that this behaviour is due to changes in the accretion flow and neutron star cooling associated with shock revival. If this behaviour is generic for exploding models enhanced GW emission may still remain a fingerprint of shock revival as it is in 2D (Murphy et al. 2009; Müller et al. 2013). With only one explosion model available to us, this conclusion does not rest on safe ground; more 3D explosion models are needed to check whether enhanced low-frequency GW emission after shock revival is indeed a generic phenomenon.
It is obviously of interest whether future GW observations will be able to discriminate between models with such distinctively different behaviour as the ones presented here. Without an elaborate statistical analysis, only limited conclusions can be drawn concerning this point. In this paper, we confined ourselves to rough estimates based on the expected excess power in second- and thirdgeneration GW detectors in two bands at low $(20 \ldots 250 \mathrm{~Hz})$ and high $(250 \ldots 1200 \mathrm{~Hz})$ frequency. Due to the reduction of the signal amplitudes compared to 3D, the prospects for second-generation detectors appear rather bleak; even the SASI-dominated models s20, s20s and s27 could not be detected out further than $\sim 2 \mathrm{kpc}$ with AdvLIGO at a confidence level of 95 percent. Third-generation instruments like the Einstein Telescope, however, could not only detect all of our models at the typical distance of a Galactic supernova ( $\sim 10 \mathrm{kpc}$ ) and strong GW emitters like s20s out to $50 \mathrm{kpc}$; the expected SNRs could even be high enough to distinguish models with enhanced low-frequency emission due to SASI from convective models based on the 'colour' of the GW spectrum. In conjunction with timing information and the neutrino signal, it may also be possible to distinguish enhanced low-frequency emission from the SASI from enhanced GW emission after shock revival as in model s20s.

However, more work is obviously needed to fully exploit the potential of GWs as a probe of the supernova engine in the case of 'ordinary', slowly rotating supernovae for which PNS convection and the SASI are the dominant sources of GW emission. Desiderata for the future include a much broader range of 3D explosion models to determine to what extent the aforementioned features in the GW signal are generic. With waveforms from longer explosion simulations, the prospects for detecting a Galactic supernova in GWs with second generation instruments may also appear less bleak than they do now based on our biased selection that includes only one explosion model evolved to $200 \mathrm{~ms}$ after shock revival.

Furthermore, it is conceivable that much more information can be harvested from the GW signals than our simple analysis suggests. Several authors (Logue et al. 2012; Hayama et al. 2015; Gossan et al. 2016) have already demonstrated the usefulness of a powerful statistical machinery in assessing the detectability of supernovae in GWs and distinguishing different waveforms (e.g. from rotational collapse and hot-bubble convection, Logue et al. 2012). Peeling out the more subtle differences between SASI- and convectiondominated models from GW signals in the face of greatly reduced signal amplitudes certainly presents a greater challenge, but thirdgeneration instruments will none the less make it an effort worth undertaking.

The GW analysis presented in this work is based on three nonrotating progenitors, and it remains to be seen whether the findings from these simulations are generic. For GW detection, it is particularly important to ascertain whether the overall reduction of the signal from SASI and convection in 3D compared to $2 \mathrm{D}$ is always as strong as in our models, where the difference is a factor of $\sim 10$. This has recently been questioned by Yakunin et al. (2017), who reported considerably higher amplitudes for a $15 \mathrm{M}_{\odot}$ progenitor than in our models and found the energy emitted in GW to be similar in their 2D and 3D simulations. Considering that we obtain weaker GW signals in 3D in models that probe a variety of different regimes, and that other 3D studies (Müller et al. 2012a; Kuroda et al. 2016) predict amplitudes in line with our findings (albeit with less rigorous neutrino transport and without a $2 \mathrm{D} / 3 \mathrm{D}$ comparison) suggests that small amplitudes $|A| \lesssim 5 \mathrm{~cm}$ are generic in $3 \mathrm{D}$ and that the strong amplitudes in Yakunin et al. (2017) are the exception rather than the norm and need further explanation. None the less, the range of 
variation in $\mathrm{GW}$ amplitude in $3 \mathrm{D}$ deserves to be explored further in the future.

There are various properties of the pre-collapse cores that will (or at least could) impact the GW signal. The influence of rotation is well known: in rapidly rotating models there is a strong GW burst associated with the rebound of the core (Müller 1982). During the post-bounce phase rotation can lead to a bar-like deformation of the core (Rampp, Müller \& Ruffert 1998; Shibata \& Sekiguchi 2005) or the development of low-mode spiral instabilities (Ott et al. 2005; Kuroda, Takiwaki \& Kotake 2014; Takiwaki, Kotake \& Suwa 2016). These flow patterns in turn lead to strong GW emission at frequencies determined by the rotational frequency. In addition, rotation can modulate processes already present in the non-rotating models, for example prompt convection or the SASI. In the models presented by Dimmelmeier et al. (2008) and Ott et al. (2012), only models with moderate rotation rates (and non-rotating models) exhibit prompt convection. The coupling between the rotation and the SASI activity can lead to an enhanced growth rate of the spiral SASI mode (Blondin \& Mezzacappa 2007; Yamasaki \& Foglizzo 2008; Iwakami et al. 2009; Janka, Melson \& Summa 2016; Kazeroni, Guilet \& Foglizzo 2016). Whether a significant proportion of supernova progenitors has moderately rotating (let alone rapidly rotating) cores is unclear. Stellar evolution models that include the effects of magnetic fields predict rather slowly rotating pre-collapse cores (Heger, Woosley \& Spruit 2005). Furthermore, the angular momentum loss due to the stellar winds seems to be underestimated by the stellar evolution models, compared to results from asteroseismology (Cantiello et al. 2014). Predictions of the initial rotation rate of pulsars, based on their current spin-down rate and age, suggest that a large fraction of the pulsar population is born with rotation periods of the order of tens to hundreds of milliseconds (Popov \& Turolla 2012; Noutsos et al. 2013).

There is also the issue of starting the simulations from spherically symmetric progenitor models. Current GW predictions like ours rely on explicitly imposed (this study) or numerical seed perturbations to trigger the development of non-radial instabilities, and it needs to be explored further whether the level of seed perturbations is partly responsible for differences in the GW amplitudes calculated by different groups (e.g. this study and Yakunin et al. 2017). Moreover, it has been found that physical seed asymmetries in the burning shells of the progenitor can influence the shock dynamics and even help to ensure a successful explosion (Burrows \& Hayes 1996; Fryer et al. 2004; Arnett \& Meakin 2011; Couch \& Ott 2013; Müller \& Janka 2015). Any change in the initial conditions that leads to a significant change in the dynamics of the supernova core should be expected to impact the GW signal. Therefore, it will be important to keep improving the predicted GW signals, in hand with the improvement of the core-collapse models.

\section{ACKNOWLEDGEMENTS}

We thank Maxime Viallet, Jerome Guilet, Gerhard Schäfer, Paul Lasky and Eric Thrane for useful discussions. At Garching, this work has been supported by the Deutsche Forschungsgemeinschaft through the Excellence Cluster Universe EXC 153 and by the European Research Council through ERC-AdG No. $341157-$ COCO2CASA. Bernhard Müller acknowledges support by the Australian Research Council through a Discovery Early Career Researcher Award (grant DE150101145). The simulations were performed using high-performance computing resources (Tier-0) provided by PRACE on CURIE TN (GENCI@CEA, France) and SuperMUC (GCS@LRZ, Germany).

\section{REFERENCES}

Abdikamalov E. B., Ott C. D., Rezzolla L., Dessart L., Dimmelmeier H., Marek A., Janka H.-T., 2010, Phys. Rev. D, 81, 044012

Abdikamalov E. et al., 2015, ApJ, 808, 70

Arnett W. D., Meakin C., 2011, ApJ, 733, 78

Bethe H. A., 1990, Rev. Mod. Phys., 62, 801

Blanchet L., Damour T., Schaefer G., 1990, MNRAS, 242, 289

Blondin J. M., Mezzacappa A., 2006, ApJ, 642, 401

Blondin J. M., Mezzacappa A., 2007, Nature, 445, 58

Blondin J. M., Shaw S., 2007, ApJ, 656, 366

Blondin J. M., Mezzacappa A., DeMarino C., 2003, ApJ, 584, 971

Brandt T. D., Burrows A., Ott C. D., Livne E., 2011, ApJ, 728, 8

Buras R., Rampp M., Janka H.-T., Kifonidis K., 2006a, A\&A, 447, 1049

Buras R., Janka H.-T., Rampp M., Kifonidis K., 2006b, A\&A, 457, 281

Burrows A., 2013, Rev. Mod. Phys., 85, 245

Burrows A., Hayes J., 1996, Phys. Rev. Lett., 76, 352

Burrows A., Hayes J., Fryxell B. A., 1995, ApJ, 450, 830

Cantiello M., Mankovich C., Bildsten L., Christensen-Dalsgaard J., Paxton B., 2014, ApJ, 788, 93

Chandrasekhar S., 1961, Hydrodynamic and Hydromagnetic Stability. Clarendon, Oxford

Colella P., Woodward P. R., 1984, J. Comp. Phys., 54, 174

Cordero-Carrión I., Cerdá-Durán P., Dimmelmeier H., Jaramillo J. L., Novak J., Gourgoulhon E., 2009, Phys. Rev. D, 79, 024017

Couch S. M., Ott C. D., 2013, ApJ, 778, L7

Couch S. M., Ott C. D., 2015, ApJ, 799, 5

Dimmelmeier H., Font J. A., Müller E., 2002, A\&A, 388, 917

Dimmelmeier H., Novak J., Font J. A., Ibáñez J. M., Müller E., 2005, Phys. Rev. D, 71, 064023

Dimmelmeier H., Ott C. D., Janka H.-T., Marek A., Müller E., 2007, Phys. Rev. Lett., 98, 251101

Dimmelmeier H., Ott C. D., Marek A., Janka H.-T., 2008, Phys. Rev. D, 78, 064056

Dolence J. C., Burrows A., Murphy J. W., Nordhaus J., 2013, ApJ, 765, 110

Epstein R., 1978, ApJ, 223, 1037

Fernández R., 2010, ApJ, 725, 1563

Fernández R., 2015, MNRAS, 452, 2071

Finn L. S., 1989, in Evans C. R., Finn L. S., Hobill D. W., eds, Frontiers in Numerical Relativity. Cambridge Univ. Press, Cambridge, p. 126

Flanagan É. É., Hughes S. A., 1998, Phys. Rev. D, 57, 4535

Foglizzo T., Scheck L., Janka H.-T., 2006, ApJ, 652, 1436

Foglizzo T., Galletti P., Scheck L., Janka H.-T., 2007, ApJ, 654, 1006

Foglizzo T. et al., 2015, PASA, 32, 9

Fryer C. L., Holz D. E., Hughes S. A., 2004, ApJ, 609, 288

Fryxell B., Arnett D., Müller E., 1991, ApJ, 367, 619

Goldreich P., Kumar P., 1990, ApJ, 363, 694

Gossan S. E., Sutton P., Stuver A., Zanolin M., Gill K., Ott C. D., 2016, Phys. Rev. D, 93, 042002

Guilet J., Foglizzo T., 2012, MNRAS, 421, 546

Hanke F., 2014, PhD thesis, Technische Universtiät München

Hanke F., Marek A., Müller B., Janka H.-T., 2012, ApJ, 755, 138

Hanke F., Müller B., Wongwathanarat A., Marek A., Janka H.-T., 2013, ApJ, 770,66

Hayama K., Kuroda T., Kotake K., Takiwaki T., 2015, Phys. Rev. D, 92, 122001

Heger A., Woosley S. E., Spruit H. C., 2005, ApJ, 626, 350

Herant M., Benz W., Hix W. R., Fryer C. L., Colgate S. A., 1994, ApJ, 435, 339

Hild S., Chelkowski S., Freise A., 2008, preprint (arXiv:0810.0604)

Hild S., Chelkowski S., Freise A., Franc J., Morgado N., Flaminio R., DeSalvo R., 2010, Class. Quantum Gravity, 27, 015003

Iwakami W., Kotake K., Ohnishi N., Yamada S., Sawada K., 2008, ApJ, 678,1207

Iwakami W., Kotake K., Ohnishi N., Yamada S., Sawada K., 2009, ApJ, 700,232

Janka H.-T., 2012, Annu. Rev. Nucl. Part. Sci., 62, 407

Janka H.-T., Müller E., 1996, A\&A, 306, 167 
Janka H.-T., Melson T., Summa A., 2016, Annu. Rev. Nucl. Part. Sci., 66, 341

Kazeroni R., Guilet J., Foglizzo T., 2016, MNRAS, 456, 126

Kotake K., Iwakami W., Ohnishi N., Yamada S., 2009, ApJ, 697, L133

Kotake K., Iwakami-Nakano W., Ohnishi N., 2011, ApJ, 736, 124

Kraichnan R. H., 1967, Phys. Fluids, 10, 1417

Kuroda T., Takiwaki T., Kotake K., 2014, Phys. Rev. D, 89, 044011

Kuroda T., Kotake K., Takiwaki T., 2016, ApJ, 829, L14,

Lattimer J. M., Swesty F. D., 1991, Nucl. Phys. A, 535, 331

Lecoanet D., Quataert E., 2013, MNRAS, 430, 2363

Lentz E. J. et al., 2015, ApJ, 807, L31

Liebendörfer M., Whitehouse S. C., Fischer T., 2009, ApJ, 698, 1174

LIGO Laboratory Shoemaker D., 2010, Advanced LIGO Anticipated Sensitivity Curves, Available at: https://dcc.ligo.org/LIGO-T0900288/public

LIGO Scientific Collaboration et al., 2015, Class. Quantum Gravity, 32, 074001

Logue J., Ott C. D., Heng I. S., Kalmus P., Scargill J. H. C., 2012, Phys. Rev. D, 86, 044023

Lund T., Marek A., Lunardini C., Janka H., Raffelt G., 2010, Phys. Rev. D, 82, 063007

Marek A., Dimmelmeier H., Janka H.-T., Müller E., Buras R., 2006, A\&A, 445,273

Marek A., Janka H., Müller E., 2009, A\&A, 496, 475

Melson T., Janka H.-T., Marek A., 2015a, ApJ, 801, L24

Melson T., Janka H.-T., Bollig R., Hanke F., Marek A., Müller B., 2015b, ApJ, 808, L42

Müller E., 1982, A\&A, 114, 53

Müller B., 2015, MNRAS, 453, 287

Müller E., Janka H.-T., 1997, A\&A, 317, 140

Müller B., Janka H.-T., 2014, ApJ, 788, 82

Müller B., Janka H.-T., 2015, MNRAS, 448, 2141

Müller E., Fryxell B., Arnett D., 1991, A\&A, 251, 505

Müller B., Janka H., Dimmelmeier H., 2010, ApJS, 189, 104

Müller E., Janka H.-T., Wongwathanarat A., 2012a, A\&A, 537, A63

Müller B., Janka H.-T., Heger A., 2012b, ApJ, 761, 72

Müller B., Janka H.-T., Marek A., 2013, ApJ, 766, 43

Murphy J. W., Burrows A., 2008a, ApJS, 179, 209

Murphy J. W., Burrows A., 2008b, ApJ, 688, 1159

Murphy J. W., Ott C. D., Burrows A., 2009, ApJ, 707, 1173

Murphy J. W., Dolence J. C., Burrows A., 2013, ApJ, 771, 52

Nakamura T., Oohara K., 1989, in Evans C. R., Finn L. S., Hobill D. W., eds, Frontiers in Numerical Relativity. Cambridge Univ. Press, Cambridge, p. 254

Nakamura T., Oohara K., Kojima Y., 1987, Prog. Theor. Phys. Suppl., 90, 1

Nordhaus J., Brandt T. D., Burrows A., Almgren A., 2012, MNRAS, 423, 1805

Nordlund Å., Stein R. F., Asplund M., 2009, Living Rev. Sol. Phys., 6, 2

Noutsos A., Schnitzeler D. H. F. M., Keane E. F., Kramer M., Johnston S., 2013, MNRAS, 430, 2281
Ohnishi N., Kotake K., Yamada S., 2006, ApJ, 641, 1018

Ohnishi N., Iwakami W., Kotake K., Yamada S., Fujioka S., Takabe H., 2008, J. Phys. Conf. Ser., 112, 042018

Oohara K.-i., Nakamura T., Shibata M., 1997, Prog. Theor. Phys. Suppl., 128,183

Ott C. D., Ou S., Tohline J. E., Burrows A., 2005, ApJ, 625, L119

Ott C. D., Dimmelmeier H., Marek A., Janka H.-T., Hawke I., Zink B., Schnetter E., 2007, Phys. Rev. Lett., 98, 261101:1

Ott C. D. et al., 2012, Phys. Rev. D, 86, 024026

Popov S. B., Turolla R., 2012, Ap\&SS, 341, 457

Rampp M., Janka H.-T., 2002, A\&A, 396, 361

Rampp M., Müller E., Ruffert M., 1998, A\&A, 332, 969

Roberts L. F., Ott C. D., Haas R., O’Connor E. P., Diener P., Schnetter E., 2016, ApJ, 831, 98

Sathyaprakash B. et al., 2012, Class. Quantum Gravity, 29, 124013

Scheck L., Kifonidis K., Janka H.-T., Müller E., 2006, A\&A, 457, 963

Scheck L., Janka H.-T., Foglizzo T., Kifonidis K., 2008, A\&A, 477, 931

Scheidegger S., Fischer T., Whitehouse S. C., Liebendörfer M., 2008, A\&A, 490, 231

Scheidegger S., Whitehouse S. C., Käppeli R., Liebendörfer M., 2010, Class. Quantum Gravity, 27, 114101

Shibata M., Sekiguchi Y.-I., 2005, Phys. Rev. D, 71, 024014:1

Takiwaki T., Kotake K., Suwa Y., 2012, ApJ, 749, 98

Takiwaki T., Kotake K., Suwa Y., 2014, ApJ, 786, 83

Takiwaki T., Kotake K., Suwa Y., 2016, MNRAS, 461, L112

Tamborra I., Hanke F., Müller B., Janka H.-T., Raffelt G., 2013, Phys. Rev. Lett., 111, 121104

Tamborra I., Raffelt G., Hanke F., Janka H.-T., Müller B., 2014a, Phys. Rev. D, 90, 045032

Tamborra I., Hanke F., Janka H.-T., Müller B., Raffelt G. G., Marek A., 2014b, ApJ, 792, 96

Thompson C., 2000, ApJ, 534, 915

Viallet M., Meakin C., Arnett D., Mocák M., 2013, ApJ, 769, 1

Wongwathanarat A., Janka H., Müller E., 2010, ApJ, 725, L106

Wongwathanarat A., Janka H.-T., Müller E., 2013, A\&A, 552, A126

Wongwathanarat A., Müller E., Janka H.-T., 2015, A\&A, 577, A48

Woosley S. E., Heger A., 2007, Phys. Rep., 442, 269

Woosley S. E., Heger A., Weaver T. A., 2002, Rev. Mod. Phys., 74, 1015

Yakunin K. N. et al., 2010, Class. Quantum Gravity, 27, 194005

Yakunin K. N. et al., 2015, Phys. Rev. D, 92, 084040

Yakunin K. N. et al., 2017, preprint (arXiv:1701.07325)

Yamasaki T., Foglizzo T., 2008, ApJ, 679, 607

This paper has been typeset from a $\mathrm{T}_{\mathrm{E}} \mathrm{X} / \mathrm{L} \mathrm{T} \mathrm{E} \mathrm{X}$ file prepared by the author. 